\title{
Imaging of Diffusion and Microcirculation with Gradient Sensitization: Design, Strategy, and Significance
}

\author{
Denis Le Bihan, MD, PhD • Robert Turner, PhD \\ Chrit T. W. Moonen, PhD • James Pekar, PhD
}

Recent developments in the use of magnetic resonance (MR) to measure and image diffusion and blood microcirculation ("perfusion") are summarized. After a brief description of the effects of diffusion and perfusion on the MR signal, the different methods (conventional spin-echo, stimulated-echo, gradient-echo, and echoplanar imaging) that have been proposed and used to image and measure diffusion and perfusion by gradient sensitization are presented, along with their advantages and limitations. The difficulties of diffusion/ perfusion imaging related to both hardware and software are then discussed. Special attention is given to specific problems encountered with in vivo studies and data analysis. Finally, the potential biologic and clinical applications are outlined, and some examples are presented.

Index terms: Blood, flow dynamics $\bullet$ Blood vessels, MR studies $17.1214 \cdot$ Brain, MR studies, $10.1214 \cdot$ Brain, perfusion • Cerebral blood vessels, flow dynamics - Cerebral blood vessels, MR studies, 17.1214 - Diffusion imaging • Echo-planar imaging • Microcirculation imaging • Physics • Pulse sequences • Tissue characterization • Treatment planning

\section{JMRI 1991; 1:7-28}

Abbreviations: $\quad$ ADC $=$ apparent diffusion coefficient, $\mathrm{CE}-\mathrm{FAST}=$ contrast-enhanced Fourier-acquired steady-state technique, CSF = cerebrospinal fluid, IVCM = intravoxel coherent motion, IVIM = intra voxel incoherent motion, $\mathrm{RF}=$ radio frequency. $\mathrm{S} / \mathrm{N}=$ signal-to-noise ratio, $\mathrm{SSFP}=$ steady-state free precession, $2 \mathrm{DFT}=$ two-dimensional Fourier transform.

\footnotetext{
${ }^{1}$ From the Diagnostic Radiology Department, The Warren G. Magnuson Clinical Center (D.L.B.) and the Biomedical Engineering and Instrumentation Program (R.T., C.T.W.M., J.P.), Bldg 10, Rm 1C660, National Institutes of Health, Bethesda, MD 20892. Received July 17. 1990; revision requested September 12; revision received and accepted September 24. Address reprint requests to D.L.B.

(c) SMRI, 1991
}

CONSIDERABLE INTEREST HAS RECENTLY been shown in the ability of magnetic resonance (MR) imaging to show and measure molecular diffusion and blood microcirculation ("perfusion"). Imaging of diffusion and perfusion holds significant promise, especially in the evaluation of anatomic and functional disorders of the brain. Diffusion and microcirculation imaging with gradient sensitization is based on the well-known sensitivity of nuclear MR to spin motion $(1,2)$. Motion thus represents a "natural" marker of the MR signal, and, theoretically, no external contrast material is needed. Diffusion and perfusion are often conceptually mixed together. However, they refer to rather different physical phenomena. Molecular diffusion is the result of the thermal, so-called Brownian, random translational motion that involves all molecules. Perfusion relates to blood delivery to tissues. and the term describes several phenomena, such as blood capillary microcirculation and blood-tissue exchanges.

Measurement of molecular diffusion may bring several potentially useful new approaches to tissue characterization and functional studies, from the determination of cell geometry to the early clinical evaluation of stroke. This interest in diffusion results from the fact that diffusion directly reflects molecular mobility. Molecular mobility also affects $\mathrm{T} 1$ and T2, but diffusion refers only to translational molecular motion, while $\mathrm{T} 1$ and $\mathrm{T} 2$ reflect complex molecular interactions involving rotational motions and exchanges ( 3 ). Moreover, T1 and T2 are MR parameters that will be affected by experimental conditions, such as the strength of the magnetic field. By contrast, diffusion is not an MR parameter; that is, diffusion can be defined outside the MR context and does not depend on the MR environment. However, MR imaging is the only in vivo technique available today for measuring diffusion directly from molecular displacements.

Noninvasive imaging of perfusion has wide and already recognized applications in tissue characterization, treatment monitoring, and functional studies. Perfusion imaging has already been performed 
Figure 1. Free versus restricted diffusion. With free diffusion, such as for water molecules in a bottle, the diffusion distance increases linearly with the square root (Sqrt) of the diffusion time (straight line) according to the Einstein equation. The slope of this straight line defines the diffusion coefficient $D$. If diffusing molecules are restricted to a closed space, such as an impermeable cell, the apparent diffusion distance to which diffusion MR imaging is sensitive depends strongly on the diffusion time. For short diffusion times, most molecules do not reach the boundaries of the medium and therefore diffusion behaves as free diffusion (ie, diffusion distance increases with diffusion time). When the diffusion time increases, more molecules reach the boundaries and are reflected back into the medium, and the measured diffusion distance "saturates" (the measured "apparent" diffusion coefficient decreases progressively to zero). If the barriers are somewhat permeable to molecules, the diffusion distance still increases with the square root of the diffusion time, but not linearly.

with non-MR imaging methods such as contrast material-enhanced computed $x$-ray tomography, which depicts blood distribution in tissues, and positron emission tomography (PET), which provides accurate blood flow measurements. The sensitivity of MR Imaging to spin motion has allowed noninvasive imaging of blood flow in large vessels (MR angiography). However, imaging and measuring blood flow at the capillary level are a considerably different challenge because the phenomena take place on a much smaller scale and the flowing component represents only a small portion of the water content in the voxel. The contribution of microcirculation to the MR signal must be distinguished from the contribution of diffusing bulk water, which may exhibit other types of microscopic motion. It may appear tempting to develop a microscopic MR angiography technique that would depict capillaries on a quasi-individual basis, but on such a small scale, diffusion effects become predominant and limit spatial resolution (4). For all these reasons, perfusion MR imaging by gradient sensitization is a difficult challenge.

A typical MR imaging voxel contains a large number of capillary segments, so that a statistical description of both capillary geometry and capillary circulation may be justified. As a result, microcirculation has been referred to as intravoxel incoherent motion (IVIM), along with other types of microscopic motion, including molecular diffusion (5). Because capillary segments are randomly oriented in each voxel, microcirculation can be described as "macrodiffusion" and one can associate with it a "pseudodiffusion" coefficient. This concept of pseudodiffusion ascribed to microcirculation perhaps explains why diffusion and perfusion, the meanings of which are so different, are often, and perhaps misleadingly, associated.

There has been some controversy on the technical issue of whether MR imaging could measure accurately such microscopic motion in an otherwise macroscopically moving environment. Diffusion or microcirculation imaging is not an easy technique.
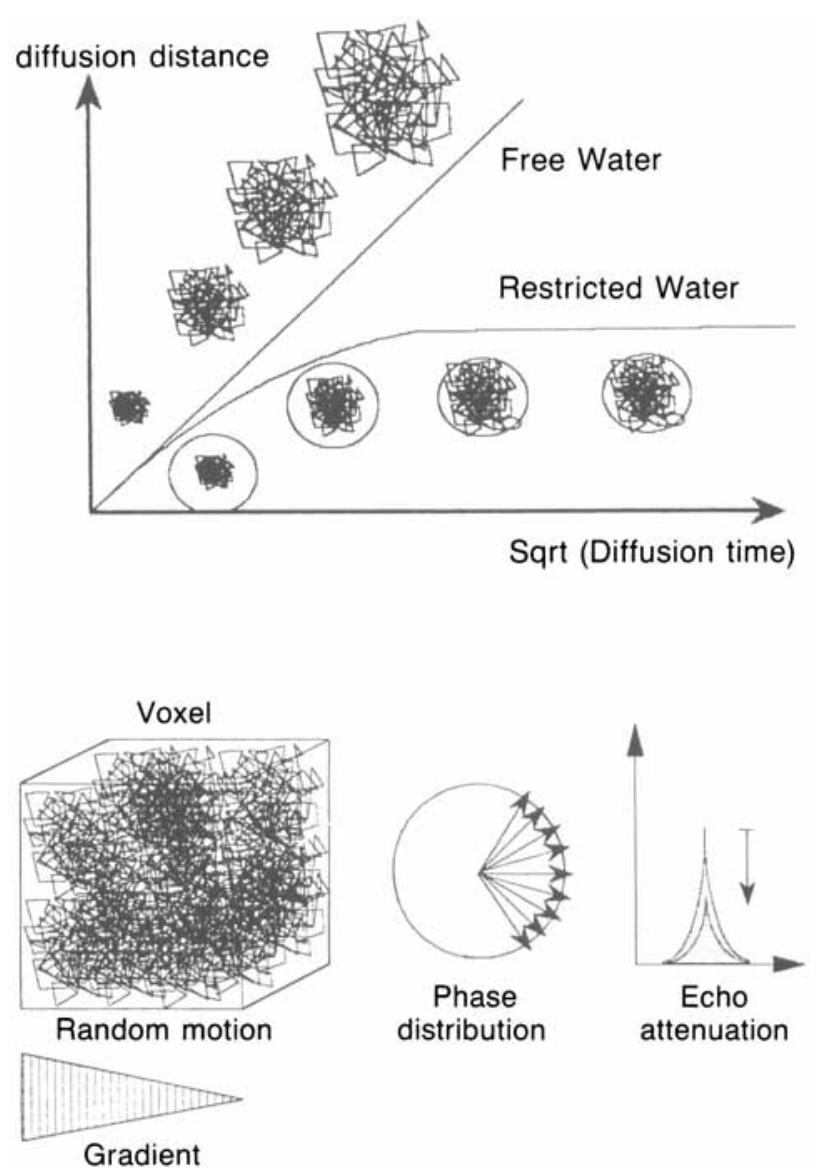

Figure 2. Random motion and MR signal. The phase of transversely magnetized spins moving in the direction of a magnetic field gradient changes compared with the phase of "static" spins, because the moving spins pass through different magnetic fields. For a population of randomly moving molecules, as in diffusion, motion along the direction of the gradient is different for each spin, resulting in a distribution of phase shifts. These phase shifts directly reflect molecular motion. In the case of diffusion. the average phase shift is zero because the average displacement is zero. The dispersion of the dephasing depends on the variance of the displacements (the square of the diffusion distance), which is related to the diffusion coefficient and the diffusion time. This dispersion of dephasings results in an attenuation of signal amplitude, which depends on the diffusion coefficient.

The many pitfalls may give rise to meaningless results, although the images may look "appealing." To obtain reliable measurements of some clinical use, the hardware and experimental setting must be carefully chosen. After a review of the different pulse sequence designs that have been proposed to image diffusion and perfusion by gradient sensitization, and a description of their main advantages and drawbacks, an important section of this article is a discussion of the strategy used and problems encountered when implementing such techniques on clinical MR imagers. Finally, the future of diffusion/perfusion imaging is discussed in terms of clinical or biologic applications. 


\section{- DESIGN: PRINCIPLES OF DIFFUSION/ PERFUSION IMAGING BY GRADIENT SENSITIZATION}

\begin{abstract}
Effect of Diffusion on the MR Spin-Echo Signal
Molecular diffusion results from a random microscopic translational motion of molecules known as Brownian motion. Because of thermal agitation, molecules are constantly moving and bouncing against each other. For a particular molecule, this random "walk" process produces net displacement over time; these displacements are randomly distributed if we consider large molecular populations. The probability that a molecule travels a distance $r$ during a time interval $t$ can be calculated. For a simple liquid, one finds a Gaussian distribution (6), the mean of which is zero, because the probability of movement in one direction is the same as that of movement in the opposite direction. The variance of the distance traveled is proportional to the time interval $t$, according to the so-called Einstein equation:
\end{abstract}

$$
\left\langle r^{2}\right\rangle=2 D t,
$$

(or $6 \mathrm{Dt}$, if we consider displacements in three dimensions). The proportionality constant $D$ is called the diffusion coeffictent and characterizes the mobility of molecules within and relative to the diffusing medium. This relation also tells us that the molecular displacement distance (diffusion distance) $r$ from the origin will increase with the square root of $t$ (Fig 1). For example, the diffusion coefficient of water at room temperature is about $2.2 \times 10^{-3}$ $\mathrm{mm}^{2} / \mathrm{sec}(7)$. This means that, during $100 \mathrm{msec}$, the standard deviation for water molecule displacements is $20 \mu \mathrm{m}$. In other words, $32 \%$ of the molecules will have a displacement greater than $20 \mu \mathrm{m}$ and $5 \%$ of the molecules will have displacements of $40 \mu \mathrm{m}$ or more. In complex systems, such as biologic systems, the distribution of molecular displacement may deviate from the Gaussian model, due to the presence of many obstacles (restricted diffusion) (8).

Diffusion can be approached in a different way, such as by using the classical Fick law (8), which models diffusion as a transport process driven by nonuniform particle (spin) concentrations. The random walk approach is, however, more suitable for explaining the effects of diffusion on the MR signal.

Diffusion is, of course, a three-dimensional process. However, the molecular mobility may not be the same in all directions. This anisotropy may be due to the physical arrangement of the medium (liquid crystal) or to the presence of obstacles that limit diffusion (restricted diffusion). In fact, diffusion is mathematically defined as a tensor rather than a scalar (9), which must be symmetric and the diagonal elements of which, $D_{x x}, D_{y y}$, and $D_{z z}$, represent molecular mobility in the three directions $x, y$, and z. (This is not a vector, since, for each axis, no privileged motion direction [ie, positive or negative] can be observed.) The nondiagonal elements, such as $D_{\mathrm{xy}}, D_{\mathrm{xz}}$, or $D_{\mathrm{yz}}$, for a medium with anisotropic diffusion tell us how diffusion in one direction corre- lates with some molecular displacements in a perpendicular direction.

Restricted diffusion occurs when molecules are confined in a limited medium by boundaries. When the molecules reach these boundaries, they are reflected back into the medium. Therefore, diffusion distance is not found to increase indefinitely with diffusion time, as seen with free diffusion, but rather "saturates" when all molecules have reached the boundaries (Fig 1). By comparison of this saturation diffusion value with the free diffusion coefficient, which can be measured with very short diffusion times (so that molecules do not experience any restriction), it is theoretically possible to evaluate the dimensions of the restrictive boundaries, of great interest for tissue characterization if the media in question are, for instance, cells. In practice, the situation is much more complicated because this size determination strongly depends on the shape of the medium (cylindrical, spherical, etc), which is not always known (8-11). Furthermore, restriction may not be complete; molecules may be able to "leak" through the boundaries, and membranes are always somewhat permeable to water $(8,12)$.

In all cases, the direction along which diffusion is measured and the time during which this measurement is accomplished are essential parameters of any diffusion experiment and must be provided with any experimental result.

The effects of molecular diffusion on spin echoes have been investigated since the early days of MR studies $(1,13,14)$. The random motion of nuclear spins carried by molecules such as water molecules, which diffuse through any magnetic field inhomogeneity, reduces the spin-echo amplitude. In the presence of a magnetic field gradient, random spin displacements produce random dephasings that destructively interfere with one another, resulting in incomplete refocusing of the echo and thus in attenuation of the echo amplitude (Fig 2). Because of the Gaussian shape of the probability distribution of diffusion displacements, this attenuation $A$ has an exponential dependence:

$$
A=\exp (-b D) \text {, }
$$

where $D$ is the diffusion coefficient (considered here as isotropic [ie, the same in all directions]) and $b$ is a factor that depends only on the magnetic field gradients. For instance, for a constant gradient $G$ applied during the echo delay TE of a spin-echo sequence, one has (1)

$$
b=\gamma^{2} G^{2} \mathrm{TE}^{3} / 12,
$$

and the echo signal intensity is represented by

$$
S=S_{0}(N, T 1) \exp (-\mathrm{TE} / \mathrm{T} 2) \exp \left(-\gamma^{2} G^{2} \mathrm{TE}^{3} D / 12\right),
$$

where $N$ refers to spin density, $\gamma$ to the gyromagnetic ratio, and $S_{0}$ would be the signal amplitude without T2 or diffusion effects. Although $b$ depends on the square of $G$ and the cube of TE, diffusion effects can be observed only with strong gradients and/or long diffusion measurement times, because $D$ is generally small in biologic tissues. For instance, in 
the case of pure water, $G$ must be as high as $0.4 \mathrm{G}$ / $\mathrm{cm}(4 \mathrm{mT} / \mathrm{m})$ to observe a diffusion-related signal loss of only $20 \%$ with a TE of 100 msec (5). Since water diffusion in tissues is less than in pure water, the attenuation will be still less.

In the early days of MR studies, field inhomogeneities due to imperfections of the magnet were large enough to produce a significant attenuation due to diffusion. To overcome this problem, Carr and Purcell suggested the use of a multiecho train rather than a single echo (13). In this case, due to the refocusing at each echo, the diffusion measurement time is split into a series of shorter diffusion times, each of which is equal to the interecho delay, and the effect of diffusion differs slightly. The $b$ value becomes

$$
b=\gamma^{2} G^{2} \mathrm{TE}^{3} / 12 n^{2},
$$

where $n$ is the echo number and TE the effective echo delay of the $n$th echo (1e, TE $=n \mathrm{TE}^{\prime}$ if $\mathrm{TE}^{\prime}$ is the interecho delay).

Owing to the factor $n^{2}$, the diffusion-related echo attenuation will be much less in the case of the multiecho sequence. Consequently, as far as diffusion effects are concerned, a single-echo and a multiecho sequence are not identical. If we want to decrease diffusion effects, the use of a multiecho train is preferable. This is especially true when measuring T2. Failure to take into account diffusion effects would lead to an underestimation of T2 (15). Diffusion measurements generally require a single-echo approach, which is more sensitive.

A significant improvement in diffusion measurements with use of a single spin echo was introduced by Stejskal and Tanner (16). With very large but short gradient pulses (hundreds of gauss per centimeter during a few milliseconds) placed on each side of the $180^{\circ}$ pulse of a spin-echo sequence (Fig 3) and balanced for "static" spins, residual $B_{0}$ field inhomogeneities become negligible in comparison with the pulses, allowing accurate measurements of very small diffusion coefficients. In this Stejskal-Tanner sequence (9), the expression for $b$ becomes

$$
b=\gamma^{2} G^{2} \delta^{2}\left(\Delta-\frac{\delta}{3}\right),
$$

where $\delta$ is the duration of each gradient pulse and $\Delta$ the time interval separating their onset (Fig 3). With the high-quality magnets of today, residual inhomogeneities are often negligible; the StejskalTanner sequence still offers an advantage in that when $\delta$ is much less than $\Delta$, the diffusion measurement time is known exactly and controllable independently of TE. The diffusion measurement time in this sequence is $\Delta-(\delta / 3)$ and can be varied. This is particularly useful for restricted diffusion studies, in which the diffusion time is a critical parameter. However, when $\delta$ is not short, as required when the gradient power available is not high, the diffusion time, although mathematically defined by $\Delta-$ $(\delta / 3)$, becomes less meaningful physically. In particular, it should be noted that the factor $\delta / 3$ comes from the integration over time of the gradient pulses (see Eq. (7]), so that this factor will be differ-

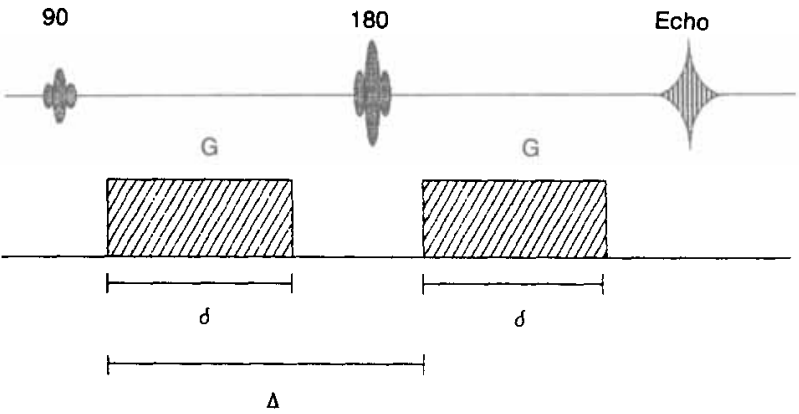

Figure 3. Stejskal-Tanner sequence. A significant improvement in diffusion measurements was introduced by Stejskal and Tanner in 1965 (16). The principle of this sequence is to use large and short pulsed magnetic field gradients instead of constant gradients, to improve accuracy. Typically, the sequence consists of two gradient pulses $G$ of short duration $(\delta)$ separated by a variable time interval $\Delta$ and disposed on either side of the $180^{\circ}$ spin-echo radiofrequency (RF) pulse.

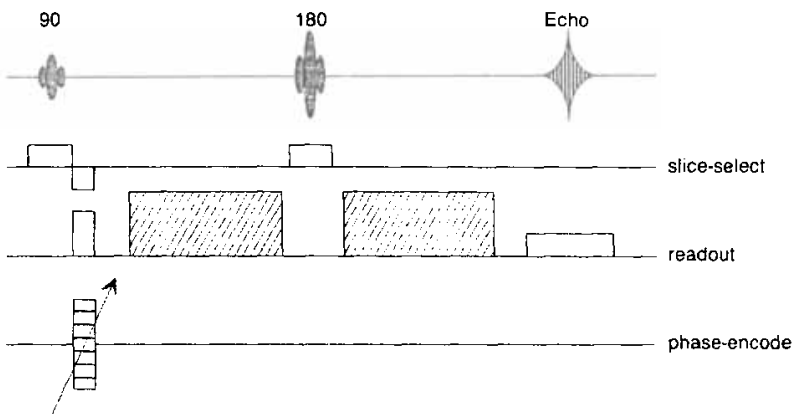

Figure 4. Diffusion imaging. Sensitization of a spinecho 2DFT Imaging sequence to diffusion can be obtained by inserting additional gradient pulses within the sequence, according to the Stejskal-Tanner scheme (Fig 3). These pulses (shaded boxes) have been set to sensitize the echo signal to diffusion along the readout gradient axis, but diffusion weighting can be obtained on any axis. By changing the amplitude and/or the duration of these gradient pulses, it is possible to modulate the degree of diffusion weighting of the echo. Diffusion images, free of $T l$ or T2 effects, are calculated by combining at least two differently diffusion-weighted images.

ent for nonrectangular gradient pulse shapes, such as sinusoidal shapes. Therefore, the diffusion time is introduced somewhat artificially to differently weight the effects of the diffusion displacements on MR signal attenuation for different times, during and between the presence of the gradient pulses.

It is desirable to avoid the presence of a gradient of large amplitude during the recording of the echo signal. Such a gradient will increase the frequency bandwith and thus reduce the signal-to-noise ratio $(\mathrm{S} / \mathrm{N})$. In the Stejskal-Tanner sequence, no gradient is required during echo sampling. If a gradient is necessary during sampling, as it is for imaging, that gradient should preferably be completely independent of the gradient pulses used for diffusion sensitization. 

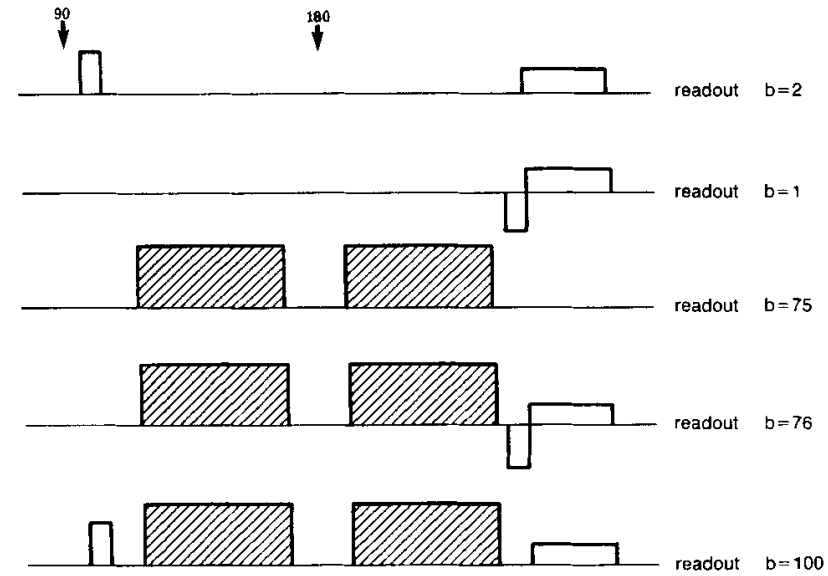

Figure 5. Effects on the gradient factor $b$ of cross terms between imaging and diffusion-sensitizing gradients. Example in which the diffusion-sensitizing gradient pulses have been set on the readout axis (as in Fig 4). RF pulses and the gradient pulses on other axes have been left out for simplicity. Units for $b$ are seconds per square millimeter. The $b$ value achieved with conventional imaging readout gradient pulses is usually negligible $(b=2)$; the $b$ value may be even further reduced by setting the predephasing and readout gradient pulses closer to each other $(b=$ 1); the $b$ value obtained from a pure Stejskal-Tanner configuration (no imaging gradients) is deliberately larger because large gradients are used $(b=75)$; the $b$ value obtained when there are no cross terms between the readout and the diffusion-sensitizing gradient pulses-because complete refocusing of static spins is achieved after the end of the second diffusion gradient and before the beginning of the readout gradient pulse pair--is equal to the sum of each contribution $(b=75+1=76)$. In the bottom example, the readout and diffusion-sensitizing gradient pulses are interleaved. This combination produces cross terms that are not negligible. The effect of the readout gradient on the $b$ value becomes important $(b=100)$, although its own contribution is small. The cross-term contribution depends on the readout gradient and will vary with the field of view, spatial resolution, and bandwith of the acquisition. For accurate diffusion determination, appropriate $b$ values must thus be calculated for each configuration by solving Equation (7), most often numerically.

\section{Principles of Diffusion Imaging}

Spin-echo sequence.-The concept of diffusion imaging is recent $(5,17-20)$. Because of the multiple pulses used in an imaging sequence, the Stejskal-Tanner equation (Eq [6]) is no longer valid and the expression for $b$ must be recalculated, taking into account all gradient pulses present in the imaging sequence (5). The general expression is

$$
b=\gamma^{2} \int_{0}^{\mathrm{TE}}|\mathbf{k}(t)|^{2} \mathrm{~d} t
$$

with

$$
\mathbf{k}(t)=\int_{0}^{t} \mathbf{G}\left(t^{\prime}\right) \mathrm{d} t^{\prime},
$$

where $\mathbf{G}\left(t^{\prime}\right)$ is $-\mathbf{G}\left(t^{\prime}\right)$ for $t^{\prime}$ greater than TE/2 (ie, after the $180^{\circ}$ pulse at $\mathrm{TE} / 2$ ).

Because of the vectorial nature of this relation, cross terms between gradient pulses placed on per- pendicular axes cancel out, since their scalar product is zero. When diffusion can be considered as isotropic, the contributions $b_{\mathrm{x}}, b_{\mathrm{y}}$, and $b_{\mathrm{z}}$ of each axis $x, y$, and $z$ can be added together, so that $b=b_{x}$ $+b_{\mathrm{y}}+b_{\mathrm{z}}$.

However, if diffusion is anisotropic, one must handle separately the different components of the diffusion tensor. If we consider diagonal terms only, the echo attenuation is

$$
S / S_{0}=\exp \left(-\sum_{i=x, y, z} b_{\mathrm{i}} D_{\mathrm{ii}}\right) .
$$

(In the presence of nondiagonal terms, Equations [7] and [9] are inexact and must be reformulated according to reference 9.)

It appears, thus, that it is inappropriate to consider, in the case of anisotropic diffusion, an "effective" $b$ factor $b_{\text {eff, }}$ such that $b_{\text {eff }}=b_{x}+b_{y}+b_{z}$. In particular, this means that if diffusion gradients are placed simultaneously on different axes, diffusion measured along the direction of the net vector gradient derived from the vectorial sum of each gradient component (ie, $G_{x}, G_{y}, G_{z}$ ) will be a complicated mixture of the diagonal and nondiagonal terms of the diffusion tensor.

For a typical two-dimensional Fourier transform (2DFT) spin-echo imaging sequence (21) (Fig 4) that contains multiple low-amplitude gradient pulses, the $b$ value remains small, typically less than $1 \mathrm{sec} /$ $\mathrm{mm}^{2}$, so that the diffusion effect is completely negligible (for $D=2 \times 10^{-3} \mathrm{~mm}^{2} / \mathrm{sec}$, the attenuation is less than $1 \%$ ) (5). To increase the sensitivity of an imaging sequence to diffusion, it is necessary to incorporate additional gradient pulses in the sequence $(5,19)$. However, the mixture of imaging and diffusion-sensitizing gradients on the same axis will produce cross terms when Equation (7) is solved to calculate $b$. These cross terms will depend on the way these gradient pulses are combined in time and may be far from negligible (Fig 5), and this is why Equation (6) cannot be applied. It is important to properly determine $b$ values by solving Equation (7), even numerically, or to design the sequence in such a way that cross terms are made negligible (Fig 5). The use of Equation (6) becomes inexact in the presence of cross terms and leads to an overestimation of the diffusion values. In the presence of such cross terms, it also appears that positive and negative diffusion gradients will behave differently. The main terms contributing to $b$ derived from Equation (7) are sign independent because they result from squared amplitude. This is not the case for the cross terms, and, therefore, the $b$ values obtained with positive and negative diffusion-sensitizing gradients will differ. By comparing the MR signals obtained with positive and negative diffusion-sensitizing gradients, or, more efficiently. by alternating positive and negative gradient pulses, one can eliminate the cross-term effects. This concept has been suggested in the past as a way to get around cross terms between diffusionsensitizing gradients and residual field gradients, which are very difficult to estimate $(22,23)$. Finally, note that in the presence of cross terms the diffu- 


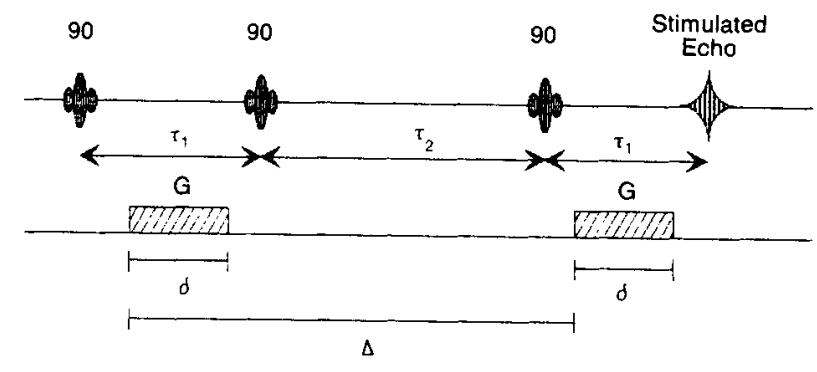

Figure 6. Stimulated-echo sequence. This sequence consists of a series of three RF pulses (in this case, three $90^{\circ}$ pulses) separated by time intervals $\tau_{1}$ and $\tau_{2}$. This combination results in several echo formations. The echo that occurs at time $\tau_{1}$ after the third pulse is called a stimulated echo. The interesting feature of this echo is that it combines T2 effects accumulated during the two $\tau_{1}$ periods and T 1 effects accumulated during the $\tau_{2}$ period. Because $\mathrm{T} 1$ is longer than $\mathrm{T} 2$ in biologic tissues, $\tau_{2}$ can be made much longer than the TE of a regular spin-echo sequence. This aids in diffusion measurements because it allows the diffusion time to be increased without increasing the amount of T2 decay. Diffusion sensitization is obtained by inserting gradient pulses $(G)$ of duration $\delta$ and separated by a time interval $\Delta$ within the $\tau_{1}$ periods.

sion time is poorly defined.

The computation of diffusion images (ie, maps on which the diffusion coefficient is displayed in each voxel) is performed with use of two or more sequences differently sensitized to diffusion. For instance, in the case of two images $\left(S_{1}\right)$ and $\left(S_{0}\right)$ obtained with gradient factors $b_{1}$ and $b_{0}$, the diffusion coefficient can be determined in each voxel from their relative signal intensities according to $(5,17)$

$$
D(\mathrm{x}, \mathrm{y}, \mathrm{z})=\ln \left[\mathrm{S}_{0}(\mathrm{x}, \mathrm{y}, \mathrm{z}) / S_{1}(\mathrm{x}, \mathrm{y}, \mathrm{z})\right] /\left(b_{1}-b_{0}\right) \text {. }
$$

Diffusion coefficients can be determined more accurately from more than two images obtained with different known $b$ values by fitting the signal attenuation $S / S_{0}$ with Equation (2).

A variety of related techniques have been proposed to image diffusion. With the spin-echo scheme, it is possible to vary the strength or the duration of the diffusion-sensitizing gradients $(24,25)$ or their direction in order to enhance anisotropic diffusion effects (26). Other schemes have been used, such as the stimulated-echo sequence (18) or variants of the steady-state free precession (SSFP) technique (27-30) and, more recently, echo-planar imaging $(31,32)$. These schemes may present some advantages when compared with the spin-echo approach and will now be discussed.

Stimulated-echo sequence.-The stimulatedecho scheme has been proposed for diffusion imaging (18). A stimulated-echo sequence consists of three RF pulses separated by time intervals $\tau_{1}$ and $\tau_{2}$ (Fig 6). This combination of three RF pulses results in several echo formations that occur at defined times. These echoes have different origins. The most interesting echo is the one called the stimulated echo, occurring a time interval $\tau_{1}$ after the third RF pulse (1).

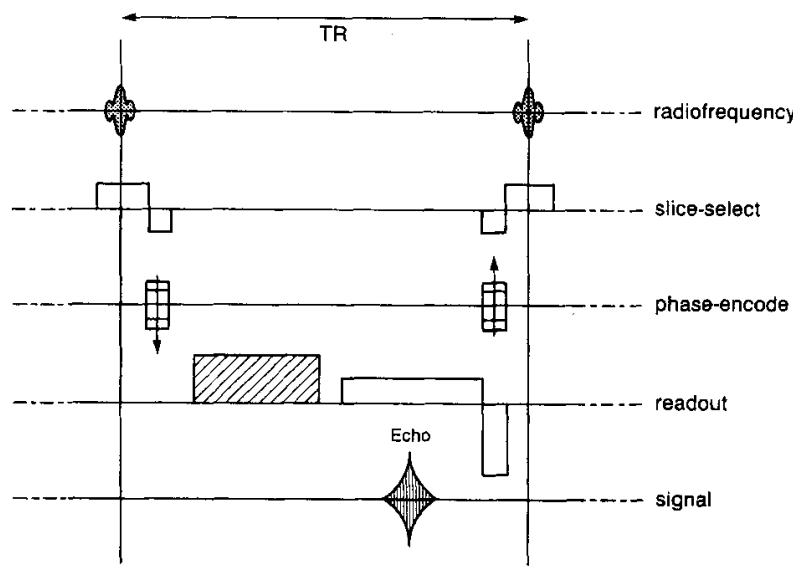

Figure 7. Diffusion imaging with SSFP. These sequences use small flip angles and gradient echoes. Depending on the design of the gradient pulses, either or both of the signal formations that occur immediately after or before each RF pulse can be selected for image acquisition. The best design for diffusion measurement is the CE-FAST scheme, in which the signal preceding each pulse is acquired to increase T2 weighting. Diffusion sensitization is obtained by inserting a single gradient pulse before the readout gradient pulse block (shaded pulse). The echo signal then has a complicated dependence on $\mathrm{T} 1, \mathrm{~T} 2$, and diffusion.

The remarkable feature of the stimulated echo is related to the magnetization evolution during the period $\tau_{2}$ between the second and third RF pulses. After the end of the second RF pulse, part of the transverse magnetization (exactly half in the case in which $90^{\circ}$ pulses are used) is stored as longitudinal magnetization. During the $\tau_{2}$ period, longitudinal magnetization thus experiences $T 1$ relaxation and not T2. Since T1 is usually much longer than $\mathrm{T} 2$ in biologic tissues, longer evolution times can be achieved than with a spin-echo sequence, without the usual signal penalty due to T2 decay.

The amplitude of the stimulated echo, in the case in which the three RF pulses are $90^{\circ}$ pulses (1), is

$$
S=S_{0}(N, T 1) / 2 \cdot \exp \left(-\tau_{2} / T 1\right) \cdot \exp \left(-2 \tau_{1} / \mathrm{T} 2\right) \text {. }
$$

This feature is particularly useful for diffusion measurement purposes when long diffusion times are required. Gradient pulses can be inserted within the first and third intervals of the stimulatedecho sequence (Fig 6). The diffusion time will include $\tau_{2}$ and can thus be longer than with a spinecho sequence (33). The Stejskal-Tanner relation (Eq [6]) still applies, provided that the period $\tau_{2}$ is included in $\Delta$. The longer diffusion time is useful for studying very slow diffusion rates or to compensate for the unavailability of large gradients. There is, unfortunately, a signal reduction of one-half when compared with the spin-echo signal, since only half of the magnetization generated at the end of the $\tau_{1}$ period contributes to echo formation. Because of $S$ / $\mathrm{N}$ considerations, it appears that this sequence is useful primarily in the presence of tissues with short T2s or when the spin-echo sequence cannot 


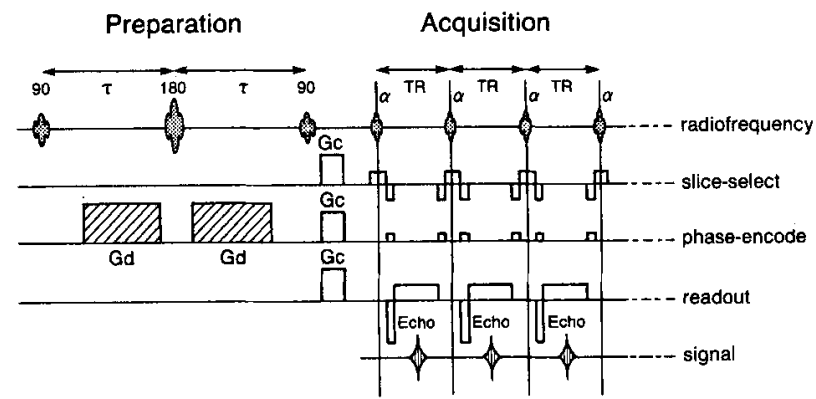

Figure 8. TurboFLASH diffusion imaging. The turboFLASH sequence consists of a series of gradient echoes produced by repeating small-flip-angle $(\alpha)$ RF pulses. Sensitization of turboFLASH Imaging to diffusion is achieved by means of a preparation sequence. The preparation scheme is a DEFT (driven-equilibrium Fourier transform) sequence: a spin-echo sequence ( $\mathrm{TE}=2 \tau$ ) in which a $90^{\circ}$ pulse has been added at the time of the echo. Therefore, the transverse magnetization, which carries the diffusion attenuation produced by the diffusion-sensitizing gradients $(G d)$, will be flipped back to the longitudinal magnet1zation axis and will serve as a starting point for the turboFLASH acquisition. The "crusher" gradient pulses $(G c)$ serve to eliminate any residual transverse magnetization after the preparation period.

be used (34). With use of a conventional spin-echo approach in tissues with short T2s, a reasonable S/N would be obtained only with short TEs. However, short TEs do not allow sufficient diffusion effects to occur, except in the presence of very large gradient pulses.

Gradient-echo sequences.-The main drawback of the previous approaches, when used in combination with a 2DFT imaging scheme, is their long total acquisition time. To get sufficient diffusion effects with conventional gradient coll sets, long TEs are required, typically $100-160 \mathrm{msec}$, so that $\mathrm{T} 2$ weighting severely impairs $\mathrm{S} / \mathrm{N}$. To overcome this problem, other ways to increase $S / N$ must be used, also at the price of long acquisition times. In practice, this means using averaging and long TRs to limit T1 saturation effects. With a TR of 1 or 2 seconds, total acquisition time is generally about 5-10 minutes. Under such conditions, only a few sets of images differently sensitized to diffusion can be acquired, limiting the accuracy of the diffusion measurement. Long acquisition times also increase the chance of patient motion, to which diffusion imaging sequences are sensitive.

The use of fast imaging techniques, therefore, appears desirable for successful imaging of diffusion. Fast imaging with gradient echoes and small flip angles based on SSFP sequences can be sensitized to diffusion with the addition of gradient pulses. However, the theoretical analysis of the effect of diffusion in such sequences is not simple $(28,29$, $35,36)$. The main problem is that $\mathrm{T} 1$ and $\mathrm{T} 2$ effects are no longer decoupled from diffusion effects as in the spin-echo sequence, in which $\mathrm{T} 1, \mathrm{~T} 2$, and $D$ contribute separately to signal attenuation in a multiplicative manner (Eqq $[4,11]$ ). The most suitable SSFP scheme for diffusion purposes is the CEFAST (contrast-enhanced Fourier-acquired steady- state technique) sequence (27-30). This sequence, which uses the echo signal occurring before each RF pulse, is heavily $T 2$ weighted and comparable to a spin-echo sequence, the TE of which is approximately twice the TR of the CE-FAST sequence. The sensitivity to diffusion is obtained by adding a gradient pulse before the readout gradient (Fig 7). Indeed, the diffusion effect that can be found is significantly larger than the effect expected from the spin-echo equivalent because of the contribution of multiple echo paths to the signal formation, as, for instance, stimulated echoes (35). The T1 and T2 contamination in the diffusion attenuation can be reasonably limited with large gradient pulses $(28,36)$. Unfortunately, when such large gradient pulses are used, the signal is highly attenuated and $\mathrm{S} / \mathrm{N}$ becomes unfavorable. Furthermore, despite its speed, this sequence remains vulnerable to motion artifacts (30), so that its benefit for diffusion imaging has not yet been demonstrated.

A different approach, based on gradient-echo fast imaging, has recently been suggested by several researchers $(37,38)$. The idea is to use a train of gradient echoes for imaging purposes only, and thereby acquisition times as short as $300 \mathrm{msec}$ can be achieved ("turboFLASH [fast low-angle shot]" [also called "snapshot FLASH"] [39]]. Sensitization to diffusion can be obtained by a preparatory sequence placed before the gradient-echo train (Fig 8). Basically, this preparation period consists of a diffusion-weighted spin-echo sequence followed by an additional $90^{\circ}$ pulse at the top of the echo, which transforms the diffusion-attenuated transverse magnetization into longitudinal magnetization, to serve as a starting point for the turboFLASH echo train. The sensitivity to motion artifacts would be significantly decreased because of the very short acquisition times. This sequence may therefore be an interesting alternative in fast diffusion imaging. However, the sequence is not immune to $T 1$ contamination that occurs during the preparation period and mainly during the echotrain acquisition, which last several hundred milliseconds: The differential diffusion effect acquired at the end of the preparation period may then be lost for tissues with short T1s because of a quick longitudinal magnetization recovery. Furthermore, the preparation scheme mimics a DEFT sequence, which requires phase cycling to cancel effects of RF field inhomogeneities, to which this sequence is sensitive (40). Similarly, it has been proposed $(41,42)$ to speed up the stimulated-echo sequènce by splitting the third $90^{\circ}$ pulse of the sequence into $n$ smaller-flip-angle RF pulses, each of them used to generate a gradient echo ("turboSTEAM") for imaging purposes. More work remains to be done to fully characterize the ability of these promising sequences to generate clean diffusion images.

Echo-planar sequence.-A major problem occurring with in vivo imaging of diffusion arises from irregular motion of the object. The sequences used are deliberately sensitized to motion by the addition of large gradients, and, hence, gross motions may lead to widely dispersed and potentially misleading artifacts. Cardiac gating has been used to 
mitigate this problem (43), but even this motion is not strictly cyclic, and random involuntary motion cannot be dealt with by this means (44). Artifacts arise from discontinuities that occur between successive cycles of a 2DFT sequence that are separated by a time interval TR that is close to the motion period. Results of such temporal incoherence are commonly visible as "ghosts" along the phaseencoding direction. These ghosts are particularly intense in the presence of diffusion-sensitizing gradients and render the diffusion measurements meaningless. Ultimately, the best way to prevent motion artifacts is to use a single-shot technique.

With echo-planar imaging, the entire set of echoes needed to form an image is collected in a single acquisition period (single shot) of 25-100 $\mathrm{msec}$ (45). For biologic motion, no discontinuity can possibly arise between successive data points, and hence there can be no motion-derived ghosting. Even if there were motion across several pixel widths during this short acquisition, only blurring and banding of the image would be likely to result. Such motion is not normally encountered in vivo if care is taken to prevent voluntary motion.

Echo-planar imaging may be easily sensitized to diffusion $(31,32,46)$ (Fig 9). For ease and convenience, the favored echo-planar imaging technique is MBEST (47), elther in its spin-echo or gradientecho form. Sensitization consists of providing a pair of large compensated gradients for an interval before rapid gradient switching and data acquisition. The refocusing may be obtained either by simply reversing the polarity of the gradient halfway through the interval over which it is applied or by inserting a $180^{\circ} \mathrm{RF}$ refocusing pulse at the midpoint (Fig 9), without reversing gradient polarity. The arrangement of the diffusion-sensitizing gradient pulses is slightly different in each case. Indeed, echo-planar imaging is just a technique to record an entire image within a single shot. Echo-planar imaging remains otherwise compatible with almost any sequence scheme, such as spin echo or stimulated echo. The echo-planar imaging switched readout gradient itself, for typical amplitudes and durations used in practice, causes a negligible loss of signal due to diffusion $(31,32,46)$.

With echo-planar imaging, motion artifacts are significantly reduced and diffusion coefficients can be determined with a higher accuracy, although $\mathrm{S} / \mathrm{Ns}$ in echo-planar images are generally less than in 2DFT images. Since the acquisition time is very short (typically less than $100 \mathrm{msec}$ ), many images differently sensitized to diffusion can be generated. Diffusion coefficients may thus be calculated from a fitting algorithm that uses all images and not just two or three. Whenever it is possible, the echo-planar imaging technique should be preferred, although echo-planar imaging is vulnerable to susceptibility artifacts responsible for image distortion or signal dropout and to chemical shift artifacts that require efficient fat suppression. Echo-planar diffusion imaging has been successfully used for measuring diffusion of water in the human brain $(32,46)$ in volunteers and patients (Figs 10-12) and may now be considered the method of reference.

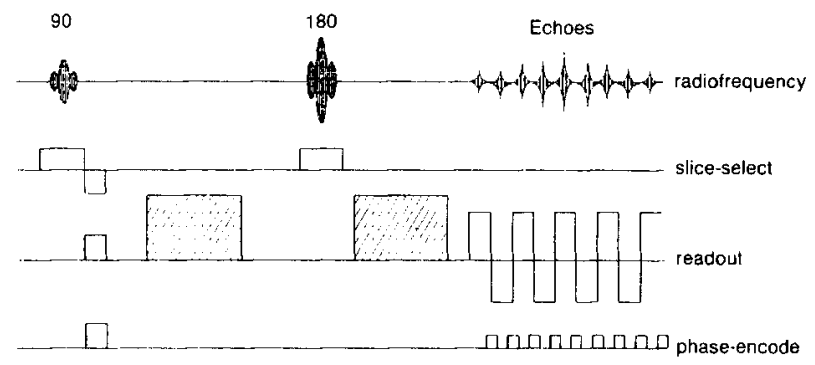

Figure 9. Echo-planar imaging of diffusion. To free diffusion images of motion artifacts, it is necessary to use fast single-shot imaging techniques such as echo-planar imaging. The Fourier space for the whole image is imaged after a single excitation pulse. In the MBEST (modulusblipped echo-planar single-pulse technique) spin-echo scheme, the spin echo is split into a series of gradient echoes by quickly switching the readout gradient. Each gradient echo corresponds to a single line of the Fourier space. Shifting from line to line is achieved by phase-encoding gradient pulses. With regard to diffusion effects, this scheme does not differ from a conventional spin-echo scheme. Diffusion sensitization results from additional gradient pulses inserted on both sides of the $180^{\circ} \mathrm{RF}$ pulse (shaded boxes).

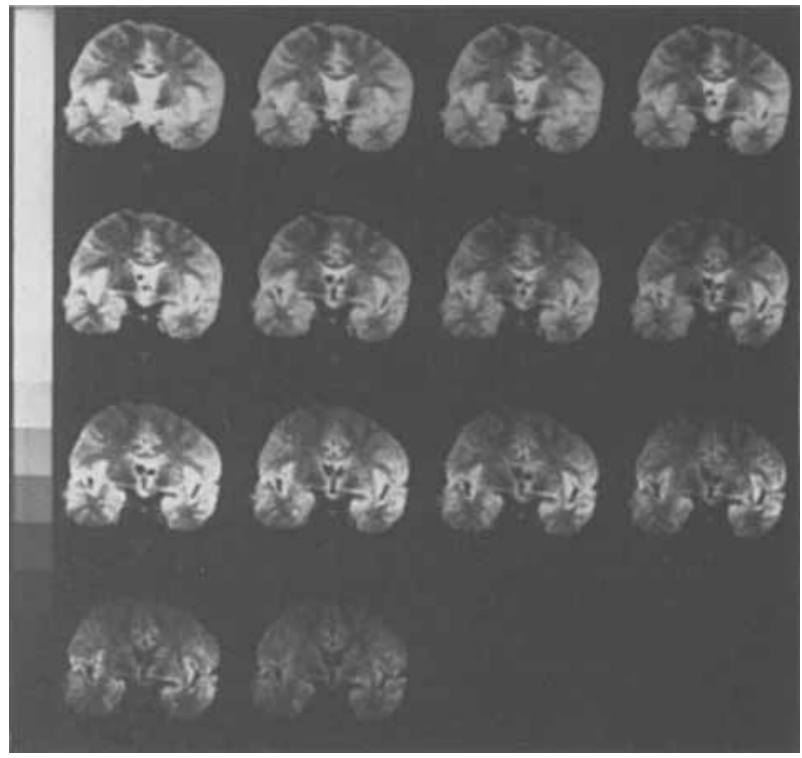

Figure 10. Study of healthy volunteer with diffusion echo-planar imaging. In this series of 14 of 16 coronal brain echo-planar images, each image has a different diffusion weighting (the diffusion gradients were varied from 0 to $3.8 \mathrm{G} / \mathrm{cm}[38 \mathrm{mT} / \mathrm{m}]$ along the $z$ axis; diffusion time $=$ $20 \mathrm{msec}$ ). Note the progressive attenuation of signal intensity as the diffusion weighting increases. Also, the degree of attenuation is clearly different for different structures, such as gray and white matter. Each image has 64 $\times 64$ pixels and was acquired in about 50 msec. In-plane resolution is $2.5 \times 2.5 \mathrm{~mm}$. and the section thickness is $10 \mathrm{~mm}$.

\section{Perfusion Imaging: Concept of Intravoxel Motion}

Effect of intravoxel motion on the MR signal.As already mentioned, spins moving in the presence of a magnetic field gradient, unlike static 


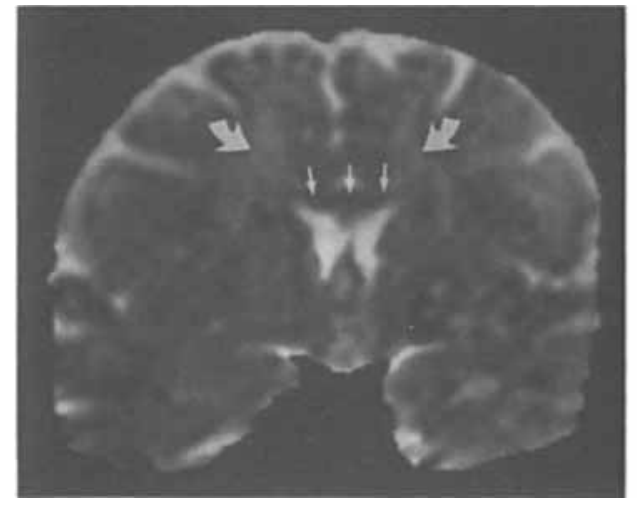

a.

Figure 11. Synthetic images derived from diffusion-weighted images. A series of 16 IVIM echo-planar coronal brain images were obtained as in Figure 10. The fit to the diffusion/ perfusion model with a nonlinear Marquardt algorithm was then performed on a pixel-bypixel basis on a Sun 3/160 workstation. (a) Diffusion image calculated from last eight images (larger $b$ values). The diffusion coefficient in each pixel is represented by means of a gray scale. Diffusion appears greater in white matter when myelin fiber tracts are parallel to the direction of the measuring gradients ( $\mathrm{z}$ axis) (curved arrows). In structures such as the corpus callosum, in which fibers are perpendicular to the measuring direction, the diffusion coefficient is small (straight arrows). (b) Initial amplitude (intercept) image calculated from the same set of data as in a. The contrast in this image is essentially T2 weighted (image equivalent to a spin-echo 4,000/130 [TR msec/TE msec] image). Note that this image looks different from the diffusion image, indicating that diffusion and T2 effects provide different information. (Reprinted, with permission, from reference 32.)

Figure 12. Echo attenuation. This plot represents the result of an experiment carried out at $1.5 \mathrm{~T}$ in a 70 -yearold human volunteer with IVIM echo-planar imaging. A sertes of 16 differently diffusion-sensitized spin-echo images were collected. The natural logarithm of the signal intensity $S$ averaged in regions of interest located in white and gray matter is plotted against the different $b$ values used for each image. For white matter, the plot follows a straight line, as expected from Equation (2) for a pure diffusion process. The slope of the straight line gives the diffusion coefficient. By using this many points, one can obtain good accuracy for the diffusion coefficient $(D=1.10 \times$ $10^{-3} \mathrm{~mm}^{2} / \mathrm{sec} \pm 0.03 \times 10^{-3}$ ). For gray matter, the plot clearly shows curvature at small $b$ values. This curvature has been ascribed to microcirculation effects, according to the IVIM model. The straight line obtained for large $b$ values gives the diffusion coefficient. The deviation from the diffusion asymptote, as measured at the intercept, gives the perfusion fraction $f$. In practice, better accuracy for $D$ and $f$ is obtained by fitting the data with Equation (18) $\left(D=1.10 \times 10^{-3} \mathrm{~mm}^{2} / \mathrm{sec} \pm 0.03 \times 10^{-3}, f=8 \% \pm 2 \%\right.$, $D^{*}=11 \times 10^{-3} \mathrm{~mm}^{2} / \mathrm{sec} \pm 6 \times 10^{-3}$ ). (The diffusion coefficient in the gray matter of this volunteer is somewhat larger than that commonly observed in younger human subjects [Table 1]).

spins, exhibit a phase shift of the transverse magnetization (48) (Fig 2). This phase shift results from changes in the magnetic field (and the associated resonance frequency) seen by spins that translate along the direction of the magnetic field gradient. The phase shift can be easily determined if one knows the translation velocity $\mathbf{v}$, the gradient amplitude $\mathbf{G}$, and the relative direction of translation at all times during the measuring sequence interval $T$, by means of the following equation:

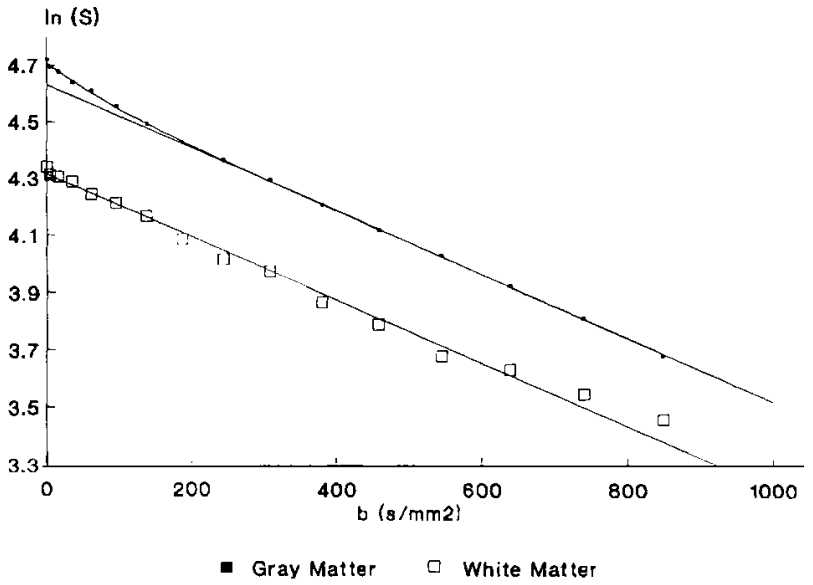

$$
\varphi=\gamma \int_{0}^{T} \nabla(t) \mathbf{G}(t) t d t
$$

No dephasing is obtained if $\mathbf{v}=0$ (no motion) or if $\mathbf{G}=0$ (no gradient), or If the direction of motion and the gradient direction are perpendicular $(\nabla G=$ $v G \cos [v, G]=0$ ). On the other hand, the effect of spin motion is a pure phase shift; in other words, the magnitude of the magnetization $\left|M_{x y}\right|$, which depends on $\mathrm{T} 1$ and $\mathrm{T} 2$, is not affected at all:

$$
M_{\mathrm{xy}}=\left|M_{\mathrm{xy}}(\mathrm{T} 1, \mathrm{~T} 2)\right| \exp (i \varphi) \text {. }
$$

The situation becomes more complicated when we consider an actual voxel in which spins may 
move independently in different directions with different velocities (intravoxel motion) $(43,49)$. An example of this is spins carried by water molecules circulating in different capillary segments of a single voxel. The effect on overall transverse magnetization in this single voxel must take into account the distribution $p\left(\varphi_{j}\right)$ of the dephasings in the voxel for each spin population $j$. A population could be represented, for instance, by all spins in a given capillary segment, which are moving with the same velocity and acquire the same dephasing. Assuming all populations have the same relaxation times, the transverse magnetization becomes

$$
\begin{aligned}
& M_{\mathrm{xy}}=\left|M_{\mathrm{xy}}(\mathrm{T} 1, \mathrm{~T} 2)\right| \sum_{j} \exp \left(i \varphi_{\mathrm{j}}\right) p\left(\varphi_{\mathrm{j}}\right) \\
&=\left|M_{\mathrm{xy}}(\mathrm{T} 1, \mathrm{~T} 2)\right| F \exp (i \Phi) .
\end{aligned}
$$

The main result is that now an amplitude attenuation $F$ may occur, reflecting the degree of incoherence of the displacements (the different dephasings within the same voxel will interfere destructively with one another), while $\Phi$ reflects the coherent part of the displacements (average net flow). Depending on (a) capillary geometry and circulation conditions and $(b)$ MR imaging acquisition parameters, the effects on $F$ and $\Phi$ may be different. Perfusion can therefore be seen as an incoherent motion $(F<1, \Phi=0$, overall amplitude attenuation in the voxel) like molecular diffusion or as a coherent motion $(F=1, \Phi \neq 0$, overall dephasing in the voxel). These two extreme conditions have been referred to, respectively, as intravoxel incoherent motion (IVIM) $(5,43$ ) and intravoxel coherent motion (IVCM) (50). A third condition would occur in the case in which the term incoherence refers to the whole voxel and not to individual spins, because only the capillary segments are randomly oriented (macroscopic incoherence). It is obvious, however, that flow may be considered coherent for individual spins, provided they keep a constant velocity within a given straight capillary segment during the time $T$ (microscopic coherence). The even-echo rephasing phenomenon can occur, so that flow-related dephasing is canceled on even echoes and there is no attenuation from flow on such echoes $(F=1$, $\Phi=0$ ). Ahn et al proposed to use this feature to map capillary perfusion (51). With this technique, two spin-echo sequences sensitized to slow flow are compared, one of them being a double-echo sequence. Another scheme would be to compare two single-echo sequences, one of which is compensated for constant linear flow (52). The order of flow compensation of the gradient nulling technique may be increased to take into consideration the tortuosity of the capillary network (53).

In all cases, the contribution of intravoxel motion constitutes only a limited, usually small, fraction $f$ of the signal originating from the voxel. Assuming there are only two components (ie, static $s$ and circulating $c$ ), with no exchanges, the total voxel magnetization is

$$
\begin{aligned}
M(\mathrm{xy})_{\text {voxel }}=(1-f) M( & \left.\mathrm{T} 1_{\mathrm{s}}, \mathrm{T} 2_{\mathrm{s}}, D_{\mathrm{s}}\right) \\
& +f F \exp (i \Phi) \mathrm{M}\left(\mathrm{T} 1_{c}, \mathrm{~T} 2_{c}, D_{\mathrm{c}}\right),
\end{aligned}
$$

where the contribution of diffusion $D$ to the magnetization magnitude $M(\mathrm{~T} 1, \mathrm{~T} 2)$ has been added.

A further drastic simplification is to consider that $\mathrm{T} 1$ and $\mathrm{T} 2$ and the diffusion coefficient of the static and the flowing component are similar, which is obviously disputable. In this case, Equation (15) simplifies to

$$
M(\mathrm{xy})_{\text {voxel }}=[(1-f)+f F \exp (i \Phi)] M(\mathrm{~T} 1, \mathrm{~T} 2, D) .(16)
$$

On the other hand, differences in $\mathrm{T} 2$ between the two components may be used to distinguish them (54). When very long TEs are used in a spin-echo sequence, the static component, which has a shorter T2, will have a decreased contribution to signal intensity compared with that of blood. This difference in T2s may also lead to an overestimation of the contributing perfusion fraction. In fact. T2 of blood is difficult to estimate because of its high sensitivity to blood oxygenation status.

\section{IVIM Imaging}

IVIM Imaging could be used to image blood microcirculation, seen as incoherent spin displacement that has a pure amplitude attenuation effect $F$, as in the case of molecular diffusion. Several models have been proposed to evaluate $F$ analytically $(43,49,51)$, but knowledge of $F$ is not required as long as we are interested only in the determination of the perfusion fraction $f$, assuming model conditions as given below.

One of these models considers microcirculation as microscopically incoherent (ie, water molecules in the capillary network frequently change direction during the measurement time $T$ ). This situation occurs when the tortuosity of the capillary network is great enough for the path of water molecules moving during $T$ to be decomposed into a series of straight segments, the length of each of which is approximately the radius of curvature (1) of the capillaries. Macroscopically, microcirculation looks like a random walk process, similar to molecular diffusion. In this idealized model, the attenuation $F$ can be easily calculated $(43,49)$ :

$$
F=\exp [-b(\langle l\rangle\langle v\rangle / 6)],
$$

where $\langle l\rangle$ is the mean free path and $\langle v\rangle$ is the mean velocity of a "flowing" blood water molecule.

The quantity $(\langle l\rangle\langle v\rangle / 6)$ has the units of a diffusion coefficient and can be called the pseudo-diffusion coefficient $D^{*}$. (Considering diffusing molecules that move at a velocity $v$ in a random walk of discrete three-dimensional jumps of magnitude $l$, one finds that the diffusion coefficient $D$ is $l v / 6$ [13].) Using literature values for $\langle l\rangle$ and $\langle v\rangle(55)$, one finds that $D^{*}$ is expected to be about 10 times larger than the true diffusion coefficient of water at room temperature. It is quite surprising to find that there is just an order of magnitude difference between $D$ and $D^{*}$, given that both phenomena occur at scales that differ by a factor of $10^{6}$. The similarity between diffusion and perfusion has allowed diffusion imaging methods to be proposed for perfusion imaging $(5,43,56)$. Moreover, the difference in order of magnitude between $D$ and $D^{*}$ should allow 
perfusion and diffusion to be distinguished on a quantitative basis (43). Determination of $D^{*}$ should give information on the capillary circulation type, combining geometry $(\langle l\rangle)$ and dynamic $(\langle v\rangle)$ properties.

Therefore, IVIM imaging is based on diffusion imaging techniques. When microcirculation effects are present, the image calculated with Equation (10) is no longer a simple diffusion image but depends also on perfusion. If it is assumed that the pseudodiffusion model is valid, the signal attenuation has a biexponential dependence on $b$ (Fig 12):

$$
S / S_{0}=(1-f) \exp (-b D)+f \exp \left[-b\left(D^{*}+D\right)\right] .
$$

Since $D^{*}$ is expected to be larger than $D$, the last points of the curve (for large $b$ values) represent diffusion almost exclusively, because microcirculation effects have been canceled out by large gradients. The first points (for smaller $b$ values) reflect both diffusion and microcirculation effects. The deviation from the diffusion asymptote (measured from the intercept of the diffusion asymptote) directly reflects the perfusion fraction $f$. It appears from this curve that a slope calculated with low $b$ values, such as $b_{0}=0$ and $b_{1}=100 \mathrm{sec} / \mathrm{mm}^{2}$, would differ significantly from the diffusion coefficient $D$ because of perfusion contamination. That is why the term "apparent diffusion coefficient" (ADC) was suggested to characterize such images calculated from two low $b$ values $(5,43)$. Obviously, the ADC is an artificial parameter that mixes diffusion and microcirculation and has no intrinsic physical significance. Nevertheless, the ADC concept has proved to be handy when perfusion is evaluated from IVIM images calculated from only two acquisitions $(5,43)$.

Characterization of signal attenuation as a function of the gradient factor $b$ would give more useful information. In particular, the three fundamental parameters-the diffusion coefficent $D$, the pseudo-diffusion coefficient $D^{*}$, and the capillary density $f$-could be separately determined by fitting the signal attenuation with Equation (18). The accurate determination of these variables requires many acquisitions with different $b$ values, which is incompatible with clinical requirements because of lengthy acquisition times when conventional 2DFT imaging techniques are used. Single-shot imaging techniques have dramatically improved this situation, allowing the attenuation curve to be routinely obtained in a clinical setting (32).

Unfortunately, determination of perfusion makes greater demands on the system than does diffusion imaging, because only a small fraction of the signal carries the microcirculation information. The $S / N$ requirement is thus much more severe and difficult to achieve. Calculation of the diffusion coefficients on a pixel-by-pixel basis with use of the last part of the attenuation curve is relatively easy with good hardware. The determination of $f$ and $D^{*}$, however, is much more difficult and usually requires the grouping of pixels into larger regions of interest in order to increase $\mathrm{S} / \mathrm{N}$ to a sufficient level, as will be demonstrated later.

\section{- STRATEGY: HOW TO SUCCESSFULLY IMPLEMENT DIFFUSION/PERFUSION IMAGING}

\section{Hardware Considerations}

There are three areas in which the demands of diffusion/perfusion imaging require special attention: gradient coils and amplifiers, field homogeneity, and design of phantoms. These will be discussed in turn.

Gradient coils and amplifiers.-Because the phase of the MR signal is proportional to the diffusion-sensitizing gradient applied, and the $b$ value is roughly proportional to the square of the gradient, it is important that the gradient be reliably characterized. To avoid excessive loss of signal due merely to transverse relaxation, it is generally better to use large gradients applied for a short time rather than weak gradients for a longer time. When gradient amplifiers are used near full power, they tend to show signs of instability such as sag or thermal cutoff, which may vary from shot to shot. In a multipulse sequence such as that used in IVIM imaging with spin echoes, any variation of the gradient from one pulse to the next introduces a discontinuity into the set of echoes that must be Fourier transformed to give the final image. Such a discontinuity manifests itself as a widely distributed image artifact.

Although such an artifact does not occur when a single-shot echo-planar technique is used in IVIM imaging, it is still necessary to have stable diffusion-sensitizing gradients, especially if data are to be averaged from the modulus images from consecutive shots. Note that it is extremely demanding on system stability to expect image phase to remain constant from shot to shot, and thus averaging in the time domain is inadvisable. For accurate diffusion/perfusion imaging it is therefore essential to have high-quality gradient amplifiers with some reserve capacity.

Not only must the diffusion-sensitizing gradients be stable, they must also be free of large eddy currents. Field gradients produced by eddy currents add to the readout gradient that immediately follows the diffusion gradients, causing readout gradients to be temporally and spatially nonuniform. This can give rise to image distortion that varies with the strength of the diffusion-sensitizing gradient, and hence can produce considerable inaccuracy in diffusion maps because of misregistration and variation in image intensity. Also, any mismatch between the intensities of the two field-gradient pulses leads to an additional damping of the echo amplitude, which is not due to diffusion, although it could be misinterpreted as such. Where short, time-constant eddy currents exist, the readout gradient may be deferred to allow their decay, but the best solution is undoubtedly to remove eddy currents at the source with use of actively shielded gradient coils (57-59), which have no fringe fields and therefore do not generate eddy currents.

Fteld homogenetty. - The $b$ value is calculated with all the gradients applied between the first $90^{\circ}$ pulse and signal acquisition. Thus, any spatial 
variation in the field gradient applied, whether due to static fleld inhomogeneity or to imperfect gradient coil design, will lead to corresponding spurious variations in the measured diffusion coefficient. Since the square of the gradient is involved, the error in the diffusion coefficient will be double that in the gradient. It is not uncommon for commercial gradient coils to produce fields that deviate from a linear gradient by as much as $5 \%$ at the edge of the field of view, which would translate into a $10 \%$ error in the diffusion coefficient. A gradient arising from static field inhomogeneity will of course be present continuously during the imaging sequence and will cause further error.

When a spin-echo (including echo-planar) diffusion sequence is used, there is yet another possible source of error: inhomogeneity in the RF field. Such inhomogeneity results in imperfect refocusing of the MR signal at the time of the echo, since the pulses will not be uniformly $90^{\circ}$ and $180^{\circ}$ across the object. There will be a residual free induction decay arising from the imperfect $180^{\circ}$ pulse, which will interfere with the spin echo and cause an artifact in the image. The normal remedy for this artifact is to use so-called crusher gradients on either side of the $180^{\circ}$ pulse, which effectively add to the diffusion gradients. However, in studying the effect of perfusion on the signal, it is at small values of $b$ (and thus at small values of the diffusion gradient) that the most change is expected. Crusher gradients can thus mask the subtle effects of perfusion, although to dispense with them creates artifact and gives unreliable results. The solution is to use an RF coil with good field homogeneity, such as a "bird-cage" coil (60). One may also improve the quality of the $180^{\circ}$ pulse by using a composite or a nonselective design or by phase cycling.

Phantom design.- Specific tests can be performed to validate the measurements before they are used in vivo. As mentioned, gradient-related artifacts may severely impair the measurements. These artifacts may not be seen when the MR system is used in a conventional way, but they may appear as soon as gradient demand is increased to perform diffusion/perfusion studies. One should look at any residual ghosts that may be related to gradient instabilities, to mechanical vibrations (the phantom must be carefully secured), or to image deformation (in particular in the direction of the diffusion-sensitizing gradients), which may result from eddy currents.

The accuracy available for diffusion measurements can be tested with a standard phantom made of liquids with known diffusion coefficients. Such liquids are water $\left(D=2.0-2.5 \times 10^{-3} \mathrm{~mm}^{2}\right.$ ) sec) (7) and acetone ( $\left.D=4.5-4.8 \times 10^{-3} \mathrm{~mm}^{2} / \mathrm{sec}\right)$ (6). These values, found in the literature, are given for "room temperature," which is not always accurately defined. Temperature-related variations in diffusion coefficients are significant, as we shall see (Fig 13), so care must be taken to control temperature during experiments. Other liquids can be used, such as glycerin, which has a very small diffusion coefficent; however, its very short T2 generally results in low signal levels. Images must be ac-

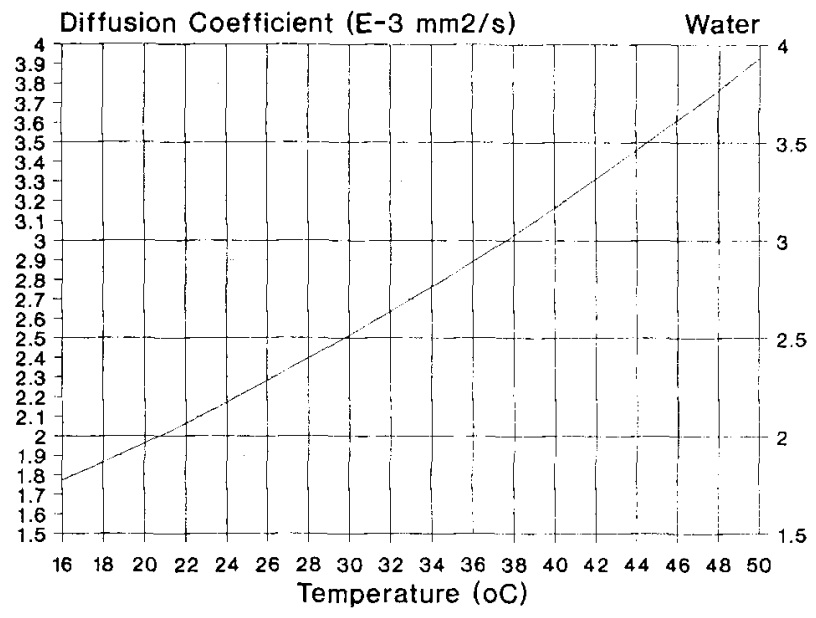

Figure 13. Relation between diffusion and temperature The relation has been well established. In the usual temperature range, there is a $2.4 \%$ change in diffusion per degree Celsius. This plot was derived partially from water diffusion measurements made on a 4.7-T MR imaging unit. The activation energy (Eq [19]) was $0.18 \mathrm{eV}, E-3=$ $10^{-3}$.

quired with different gradient intensities (ie, different $b$ values) to plot the relationship between $\ln (S)$ and $b$. By doing so, one can check the degree of scatter among measured values, reflecting the accuracy of the measurements. The slope in the plot of $\ln (S)$ versus $b$ gives the diffusion coefficient (see Eq [10]).

The use of such a phantom may not be completely adequate for in vivo studies because only a limited number of diffusion values are available. It might be suitable to have a continuous range of diffusion coefficients, especially in the range expected in vivo. A possible way to do so is to design a gel phantom in which a temperature gradient can be induced (25) (eg, by using hot and cold water streams). Temperatures can be monitored within the gel with a series of miniature probes. The relationship between diffusion and temperature is well established for water $(6,7,61)$ (Fig 13):

$$
D=D_{0} \exp \left(-E_{a} / k T\right),
$$

where $k$ is the Boltzmann constant and $E_{\mathrm{a}}$ the activation energy. This activation energy corresponds to the energy required to break two hydrogen bonds $\left(E_{\mathrm{a}}=0.18 \mathrm{eV}\right.$ ) (62). The sensitivity of diffusion to temperature is about $2.4 \%$ per degree kelvin. A diffusion image of this gel should exhibit a diffusion coefficient gradient along the direction of the thermal gradient, with greater diffusion in warmer regions. The diffusion coefficient can then be fitted to Equation (19) and used to calibrate the system.

For evaluation of the effect of perfusion, a specialized phantom must be used. To model tissue in which capillaries are randomly positioned and oriented, a chromatographic column packed with Sephadex polyacrylamide gel has been successfully used (43). This resin is made of porous microspheres through which water diffuses. The motion of water flowing between them is for the most part 
incoherent, although a net flow obviously exists. The size of the microspheres can be chosen so that the geometric microscopic disposition of the resin mimics biologic issue. Other materials have been suggested, such as cotton, sand, or glass balls, but it is difficult to control the homogeneity of the phantom, and susceptibility effects may impede the measurements. It has also been proposed that excised but perfused organs, such as kidneys, be used (63).

\section{In Vivo Systems}

When the diffusion imaging technique is applied in vivo, additional problems may occur. These are related to motion artifacts and to the distinguishing of blood circulation. Furthermore, possible restricted or anisotropic diffusion effects require special care in the experimental design.

Motion artifacts are mainly a concern for diffusion imaging sequences derived from the 2DFT scheme, in which motion-related dephasing may differ from view to view and cause ghosting. However, even with single-shot techniques, such as echo-planar imaging, care must be taken in eliminating voluntary motion. Animals must be anesthetized. Human volunteers and patients must be comfortably secured within the magnet. Use of cushions or inflating devices have been suggested. However, the main problem is internal motion due to respiration and cardiac motion. This motion has prevented successful diffusion imaging in the body. (Diffusion imaging in parts of the body may become feasible with echo-planar imaging [64].) Diffusion imaging in the extremities and the brain is much easier, but some precautions are required before assessment of measurements. It is known that the brain is subject to pulsations generated by large blood vessels (65). Similarly, there is cerebrospinal fluid (CSF) flow in the ventricular cavities of the brain. The variation of these motions from view to view may be large enough to produce significant artifacts in the presence of diffusion-sensitizing gradient pulses, even with cardiac gating, making measurements meaningless. It is, furthermore, not always easy to determine the optimum delay time between cardiac beats and signal acquisition that will minimize motion artifacts. It may also be necessary to skip every other beat to increase TR to get an increased $\mathbf{S} / \mathrm{N}$. Cardiac gating is often useful, but cardiac motion is not strictly cyclic. Besides phase effects, instabilities in the cardiac rate will induce artifacts when TR is not long compared with the $T 1$ values of the imaged tissues because of variations in saturation effects in longitudinal magnetization between successive cycles.

To decrease the chance of misregistration (and to circumvent possible system drifts), it is generally useful to interleave the differently sensitized acquisitions. Motion-compensated sequences may also be used (52). In such sequences, each of the diffusion-probing gradient pulses is bipolar to cancel dephasings produced by coherently moving spins. Unfortunately, the succession of positive and negative gradient pulses required for flow-compensation purposes considerably reduces the value of the gra- dient factor $b$, so that very large gradient pulses are needed to measure the same diffusion effects. Furthermore, first-order motion compensation cancels motion artifacts only for spins moving with a constant velocity. This kind of approach, however, has the potential to distinguish between two types of microcirculation (microscopically coherent and incoherent), because no refocusing can be obtained for completely incoherent motion. In any case, the best solution would be to use single-shot techniques such as echo-planar imaging. An interesting alternative, however, if echo-planar imaging is not available, is to use a single-shot line-scan technique that does not use phase encoding (44). Images are reduced to a band, but the measurements are more reliable.

Another specific problem encountered in vivo relates to microcirculation effects. As has been explained, perfusion may mimic diffusion (pseudodiffusion). Fortunately, the signal attenuation from perfusion is expected to be more pronounced than that due to diffusion because $D^{*}$ is greater than $D$, so that the signal coming from flowing blood is canceled out when $b$ values as low as $100-200 \mathrm{sec} /$ $\mathrm{mm}^{2}$ are used. Diffusion measurements, a priori free of perfusion contamination, therefore require measurement with larger $b$ values.

As mentioned, restricted diffusion is one of the most intriguing questions regarding in vivo diffusion measurements. Physically, restricted diffusion means that measured diffusion coefficients depend on the diffusion time made avallable to molecules. When performing a diffusion coefficient measurement, one should be careful that the diffusion time remains constant for the images used to calculate this coefficient. Methods in which the gradient pulse intervals or durations are varied are thus precluded. The correct way to measure the diffusion coefficient is to change only the gradient amplitude. However, it is of great interest to be able to change the diffusion time in order to see restricted diffusion effects. In this case, the new diffusion time has to be constant for all points in a given set of points that will be used to calculate diffusion. Similarly, anisotropic diffusion necessitates that the gradient pulses used to calculate a diffusion coefficient are on a given axis and not mixed on several axes. Measurements can then be repeated with gradient pulses applied in another direction to evaluate anisotropy.

We can conclude that the experimental setting may greatly influence the measurements. Consequently, for purposes of comparison of measurements, all investigators should provide information such as temperature, gradient direction, gradient timing within the sequence, and so forth. The calculated $b$ values must be checked with established phantoms to rule out any problem related to miscalibration or hardware.

\section{Data Analysis Algorithm}

The final step in IVIM imaging is the creation of spatial maps of the IVIM parameters from the initial "IVIM-weighted" MR images. This includes the creation of diffusion images (maps of the apparent 
diffusion coefficient) and the mapping of perfusion by measurement of the perfusion fraction at each pixel.

For diffusion imaging, two methods have been used to calculate the diffusion constant from the experimental decay of signal $S$ versus gradient factor $b$. These are (a) linear regression algorithms (fitting a straight line to the logarithms of the signal) and $(b)$ nonlinear iterative fitting algorithms (fitting an exponential decay to the actual data). The linear regression approach may have a better calculational efficiency, provided errors are transformed so that they are properly weighted (66). Otherwise, the iterative approach might be preferred, since it directly weights the data points correctly.

Similarly, to obtain an estimate of the perfusion fraction from the experimental decay of $S$ versus $b$, two methods have been used that we call asymptotlc fitting and full fitting. Asymptotic fitting involves fitting a straight line to the diffusional asymptote (the first term in Eq [18]) and then extrapolating to the intercept of this line at $b=0$. The ratio between the extrapolated intercept of the diffusional asymptote and a single measurement made with $b=0$ gives an estimate of the perfusion fraction. The pseudo-diffusion coefficient $D^{*}$ (perfusion) is not determined. Furthermore, this approach is sensitive to the choice of when the asymptote has been reached and to the low $\mathrm{S} / \mathrm{N}$ of the asymptotic points.

Full fitting, on the other hand, requires leastsquares fitting of the perfusion/diffusion decay to Equation (18). This fitting is typically done with the Marquardt iterative nonlinear least-squares method $(66,67)$ to refine a "starting guess" based on curve stripping. All four parameters of the model $\left(M_{0}, D, D^{*}\right.$, and $\left.f\right)$ are estimated from the measured data, along with estimates of their statistical uncertainties.

Monte Carlo simulation has been used to assess the accuracy of IVIM parameter estimation from noisy data. Monte Carlo methods involve use of a computer to play a game of chance to help solve a mathematical or physical problem of interest (68). A computer is used to contaminate "ideal" data with noise and to fit the resulting noisy data to give estimates of the IVIM model parameters. Repetition of this procedure, with use of many different noise sets, provides a distribution of parameter estimates. This distribution of values indicates the intrinsic uncertainty of the measurement at a given noise level.

The performance of asymptotic and full curve fitting to IVIM decays was studied with this approach (69). Results for the diffusion coefficient are shown in Figure $14 \mathrm{a}$, which plots the standard deviation of the estimated diffusion constant versus S/N. Even for the smallest $S / N$ considered $(S / N=40)$, measurement of the diffusion coefficent is still accurate to within $20 \%$. Figure $14 \mathrm{~b}$ plots the standard deviation in the estimated perfusion fraction versus $\mathbf{S} / \mathrm{N}$. Determination of the perfusion fraction to within $20 \%$ requires data with an $\mathrm{S} / \mathrm{N}$ of close to 400. From the Monte Carlo simulations, two main conclusions can be drawn: (a) Estimation of the dif- fusion constant is feasible even at rather small S/ Ns, and $(b)$ large $S / N s$ are required for accurate estimation of the perfusion fraction. These large $\mathrm{S} / \mathrm{Ns}$ are likely to be achievable only through averaging over larger regions of interest in the original IVIMweighted MR images.

Another point resulting from Monte Carlo simulations is that the $\chi^{2}$-based estimates of the uncertainty in the perfusion fraction are likely to seriously overstate the accuracy of the measurement, because for small S/Ns the estimated standard deviations are not normally distributed.

Finally, when fitting data with the IVIM model, one should carefully evaluate the likelihood of the validity of the assumptions behind the model in order to predict the size of the errors that are made in the estimation of the IVIM parameters. Among these hypotheses are that $D, T 1$, and $\mathrm{T} 2$ are similar in the static and flowing components and that exchanges between these components are slow at the MR imaging experiment scale. The latter assumption is reasonable in brain (70); however, the most critical hypothesis here is the similarity of T2 in tissues and in flowing blood (one can always work under conditions that prevent $T 1$ saturation). If the T2 of blood is much larger than the T2 of tissues, the perfusion fraction $f$ is overestimated (43), especially when long T2s are used. This may explain why the preliminary values of $f$ found in the literature are often larger than expected from anatomic data. On the other hand, T2 in blood may vary widely with the blood oxygenation state. The determination of $D$ is not, however, affected by differences in T2s, since the perfusion contribution to the signal is negligible for large $b$ values.

With the assumption that some macroscopic coherence is found in perfusion flow, so that a net flow can be detected in the voxel, the average flowrelated dephasing in the voxel differs from zero. In this case, phase-sensitive reconstruction processes may allow access to perfusion, as suggested by Young et al (50). In practice, to generate IVCM images, the data obtained from IVIM imaging methods have to be processed so that phases rather than amplitudes are used. Quantification of flow is difficult to obtain, however, because calculation of the average dephasing in a given voxel depends not only on the dephasing produced by the fraction of coherently moving spins but also on the fraction of incoherently moving spins and the fraction of static spins that are not dephased. In most cases, static spins will predominate, so the overall voxel dephasing does not accurately reflect the dephasing produced by the flowing component. The contribution of the static component may be reduced by using long TEs.

Perhaps more interesting is that, depending on the direction of the gradient pulses used, the direction of the average flow in a voxel can in principle be determined. Indeed, it could be possible to consider blood velocity as a separate dimension in the Fourier domain and to evaluate the velocity distribution within the voxel (71). Again, the data obtained with the IVIM method can be used. The IVIM data have to be Fourier transformed against the 


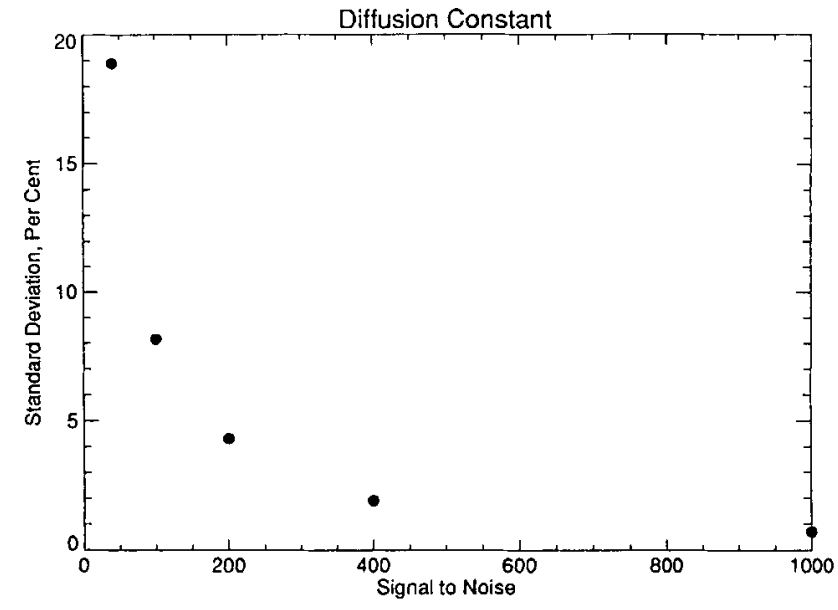

a.

Figure 14. (a) Results of Monte Carlo simulations: standard deviation in estimated diffusion constant versus S/N of first experiment; $b=10,20,30,40,50,60,70,80,90,100,200,300,400,500,600$, and 700 . This range of $b$ values should allow extraction of perfusion parameters. Values of the perfusion/diffusion model parameters: initial amplitude $M_{0}=1.0$, perfusion fraction $f=5 \%$, diffusion constant $D=1 \times 10^{-3} \mathrm{~mm}^{2} / \mathrm{sec}$, and $D^{*}=10 \times 10^{-3} \mathrm{~mm}^{2} / \mathrm{sec} ; 10,000$ repetitions were used to determine the standard deviation at each S/N. (b) Results of Monte Carlo simulations: standard deviation in estimated perfusion fraction versus $\mathrm{S} / \mathrm{N}$ of first experiment; same parameters as in a.

\begin{tabular}{|lc|}
\hline $\begin{array}{l}\text { Table } 1 \\
\text { Diffusion Coefficients in Euman Brain }\end{array}$ \\
\hline \multicolumn{1}{c}{ Tissue } & $\begin{array}{c}\text { Diffusion Coefficient } \\
\left(\times 10^{-3} \mathrm{~mm}^{2} / \mathrm{sec}\right)\end{array}$ \\
\hline CSF & $2.94 \pm 0.05$ \\
Gray matter & $0.76 \pm 0.03$ \\
White matter & $0.22 \pm 0.02$ \\
Corpus callosum & $1.07 \pm 0.06$ \\
Axial fibers & $0.64 \pm 0.05$ \\
Transverse fibers & \\
\hline
\end{tabular}

Note.-Data were obtained from volunteers by means of echo-planar imaging. Orientation of myelin fibers in white matter is defined relative to the direction of the measuring gradient pulses, which in this case were along the $B_{0}(z$ axis) direction.

\section{Table 2}

Difusion Coefficients in Some Tissues Normalized to that of Water

\begin{tabular}{ll}
\hline Tissue & $D_{\text {tissuc }} / D_{\text {water }}$ \\
\hline Muscle (77) & $0.61^{*}$ \\
Muscle (77) & $0.44^{+}$ \\
Liver (8) & $0.25-0.30$ \\
Brain (72) & $0.45^{+}-0.10^{8}$ \\
Heart (8) & $0.34-0.37$
\end{tabular}

Note.-Data from the literature, obtained ex vivo in rats at room temperature.

- Measurement parallel to muscle fibers.

- Measurement perpendicular to muscle fibers.

* 20-msec diffusion time.

${ }^{8} 60$-msec diffusion time.

successive values of the diffusion-sensitizing gradient pulses. Here also, many differently sensitized acquisitions are necessary to obtain reasonable resolution of velocities, making IVIM-echo-planar
Imaging the most suitable sequence for such studies.

\section{- SIGNIFICANCE: USEFULNESS OF DIFFUSION/PERFUSION IMAGING}

Tissue characterization may benefit greatly from molecular mobility measurements. Although water is a common and simple molecule, useful information can be found by studying its mobility $(62,72)$. The diffusion coefficient of water in tissues is two to three times less than its value when pure (Tables $1,2)$. This is largely explained by the high viscosity of bulk water in tissues due to the presence of large molecules such as intracellular proteins, to which water molecules are partially bonded. Tissues (normal to abnormal) with different viscosities or a different balance between intra- and extracellular water will have different diffusion coefficients, the source of contrast in diffusion images.

The diffusion range of water during typical MR diffusion times (100 msec) is on the order of a few micrometers, which is in the size range of many biologic structures, such as cells. Water diffusion will thus be a useful marker of tissue structure at the microscopic level, which is much smaller than the resolution of an MR image (typically about $1 \mathrm{~mm}$ ). For instance, the presence of obstacles to diffusion (eg, cell walls, membranes, fibers, or intracellular organelles) results in measurable impeded or restricted diffusion effects; because of the reduced range of possible displacements or the tortuous pathways, the diffusion coefficient is reduced compared with that of free water (Fig 1). Diffusion, therefore, allows the study of compartments much too small to be observed directly with conventional MR imaging methods (8-12). This reduction becomes more prominent in the case of restricted diffusion when diffusion times are lengthened, since an increasing fraction of molecules will strike diffu- 
sion barriers during the measurement period. This effect has been shown in plant tissues, in which the cell wall is essentially impermeable to water transport (73), or by measurement of diffusion of metabolites that remain inside the intracellular compartment (74). In living animal tissues, however, cell membranes are permeable to water (75), and there are rapid exchanges among different compartments such as intra- and extracellular spaces (76). Therefore, diffusion is more likely to be "impeded" by random obstacles such as macromolecules than to be truly "restricted" by barriers.

Furthermore, since hindrance or restriction may not be the same for different directions of motion. the measured diffusion coefficients may vary according to the direction of measurement (anisotropic diffusion). Examples have been shown in muscle (77) and more recently in cat (26) and human $(32,44,78)$ brain white matter. Water diffusion in gray matter does not exhibit anisotropy $(26,78)$ or restriction $(78,79)$ by impermeable walls. By contrast, diffusion in white matter is apparently extremely variable (Table 2). This large variability in white matter diffusion may only reflect anisotropic diffusion, measurement results depending on the respective orientation of the myelin fiber tracts and the gradient direction at each imaging location. It appears that diffusion coefficients are significantly decreased when the myelin fiber tracts are perpendicular to the direction of the magnetic field gradient used to measure molecular displacements. A simple model would be the restriction of water displacements across axon diameters in the axonal spaces by the axon membrane-myelin sheath complexes. When diffusion measurements are parallel to the direction of the fibers, diffusion is much less restricted, resulting in larger measured diffusion coefficients. In reality, the situation is more complex, because it has been shown that the myelin sheath is somewhat permeable to water $(75,78)$. Recent experiments in human white matter have demonstrated that axonal water can diffuse across myelin at a slow rate but without restriction (78). In particular, no saturation of the diffusion distance has been found when the diffusion time is increased, as would be expected for restricted diffusion (Fig 1). The reduced value of the diffusion coefficient across myelin fibers could thus reflect only a decreased water mobility through the successive lipid layers (78) (Fig 15). On the other hand, the enhanced value of "diffusion" measured parallel to the axoplasm could arise from facilitated transport favored by the highly oriented intraaxonal microstructures (ie, microtubules or microfilaments) in relation to axoplasmic transport. This transport is not a diffusional process but could be seen as an incoherent motion at the voxel scale. The measurement of anisotropic diffusion in white matter offers exciting potential applications. Myelin fiber orientation mapping may be useful for better understanding of white matter diseases such as multiple sclerosis or abnormal white matter development in children.

Recent progress made in in vivo MR spectroscopy allows the extension of diffusion measurements to

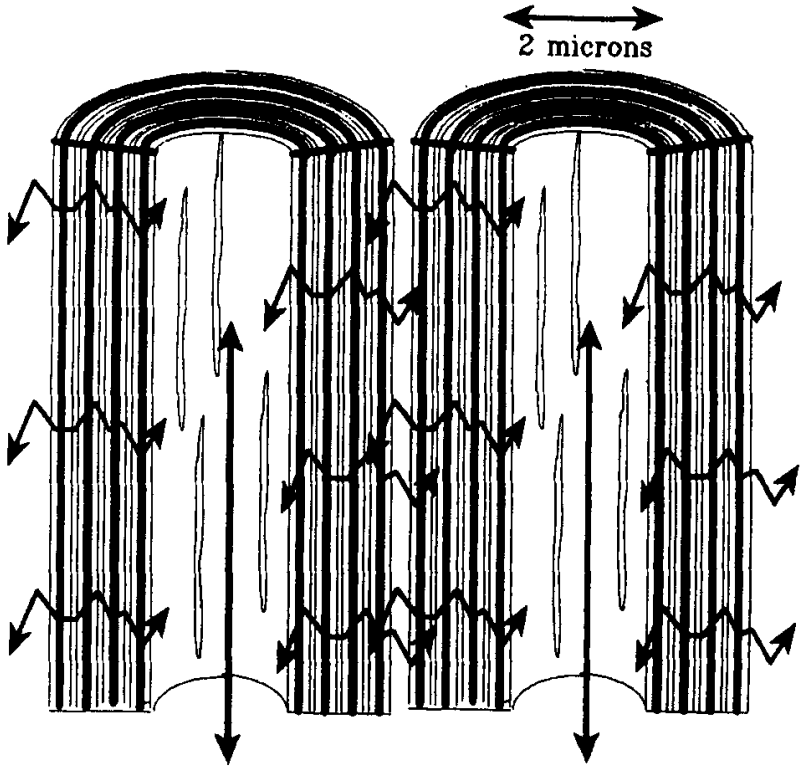

Figure 16. Anisotropy of diffusion in myelin. Diffusion in white matter has been found to be highly anisotropic (Fig 11). The diffusion coefficient is larger when measurements are obtained with the gradient parallel to the myelin fibers $\left(D=1.2 \times 10^{-3} \mathrm{~mm}^{2} / \mathrm{sec}\right)$ than when it is perpendicular $\left(D=0.4 \times 10^{-3} \mathrm{~mm}^{2} / \mathrm{sec}\right)$. One possible explanation is that water is more free to move along the axon fibers. Furthermore, diffusion of water in this direction could be facilitated by axoplasmic flow mechanisms. Diffusion perpendicular to the fibers may be impeded by the succession of myelin layers, which have a low permeability to water.

molecules other than water. Phosphocreatine, for instance, which can be studied with phosphorus31 spectroscopy (74), is a true intracellular probe, in contrast to water, which diffuses across cell membranes. Phosphocreatine (or perhaps $\mathrm{N}$-acetylaspartate in neurons) may be used to provide exclusive information on the intracellular medium, such as viscosity or geometry. Monitoring of exchanges of metabolites or drugs through cell membranes could also benefit from similar techniques designed to measure molecular flow (80).

However, so far, only much more modest results have been obtained with diffusion imaging in a clinical context $(5,43,81$ ) (Figs $16-18$ ). The best-established result is that diffusion coefficients are significantly larger in structures in which diffusion is free, such as cysts (in which the diffusion coefficient is close to that of pure water, depending only slightly on the viscosity of cystic fluid). This feature becomes clinically useful when, owing to a high paramagnetic protein content, $\mathrm{T} 1$ and $\mathrm{T} 2$ are not as long as would be expected for a liquid, so that some complicated cystic lesions have the same $\mathrm{T} 1$ and T2 appearance as that of a solid tumor (82). Diffusion imaging clearly demonstrates in these cases the liquid nature of the lesion (5). Because of the large difference in ADC between solid tumors, such as epidermoid tumors, and pulsatile cisternal 

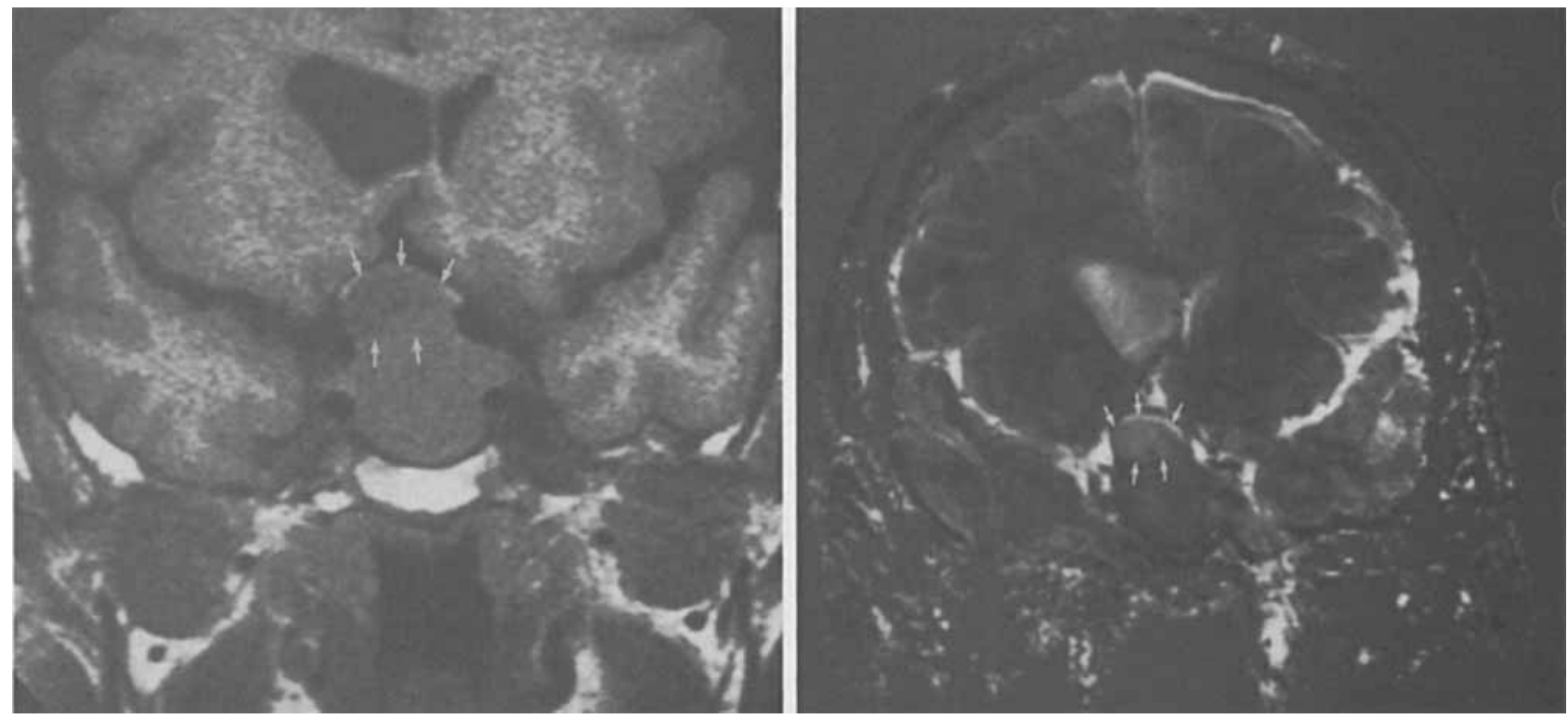

a.

b.

Figure 16. Cystic pituitary macroadenoma. (a) Coronal T1-weighted image (400/26) shows the pltuitary tumor with an area of slightly increased Tl (lower signal intensity) at its superior pole (arrows). However, T1 is shorter than expected for a liquid (CSF appears completely dark with this sequence), so that the nature of this part of the tumor (solid or liquid) cannot be determined. (b) IVIM image of the same section. The cystic nature of the superior pole of the tumor (arrows) is strongly suggested by its large ADC value, similar to the diffusion coefficient of free water as found in the nearby ventricular cavities. (In some parts of the ventricles, the ADC is even larger because of CSF flow.) The solid part of the tumor beneath the cyst has a much smaller ADC, similar to that of brain parenchyma. Distinguishing solid from cystic lesions with diffusion imaging is more rellable than with $\mathrm{T} 1$ - or $\mathrm{T} 2$-weighted imaging because diffusion is insensitive to the presence of proteins or other paramagnetic substances in the cyst liquid. Such substances may alter T1 and T2 and cause cysts to have the appearance of solid lesions.

CSF or even static fluid collections, IVIM imaging has been useful in improving conspicuity, specificity, and therapeutic management of extraaxial brain tumors (81). Furthermore, IVIM imaging is highly sensitive to incoherent CSF motion and has been proposed as a means to study CSF flow in the brain and spine $(5,83)$.

Perhaps more promising is the ability of diffusion or, more generally, IVIM imaging to detect stroke at a very early stage. It has recently been shown by diffusion imaging that the diffusion coefficient of water is significantly decreased within minutes after an ischemic insult, while all other imaging techniques, including conventional MR imaging, fail to detect any change (84). This decrease in diffusion could reflect a modification of the water balance in the tissue and a viscosity increase, possibly due to the massive entry of ions and accompanying water into the intracellular space as a result of the opening of calcium channels and the failure of ionic transmembrane pumps (cytotoxic edema) $(84,85$ ) (Fig 19). At a later stage (subacute ischemia), when conventional MR images become abnormal (showing an increase in T2), the diffusion coefficient increases well above its normal value (86); this increase is associated with vasogenic edema in which the motion of bulk water is known to be an imporant factor. Diffusion imaging thus offers the unique opportunity to address, noninvasively and in a clin- ical setting, fundamental issues regarding the response of brain tissue to stroke at different stages, with potentially important clinical implications. Early detection of stroke, at a stage when tissue damage is still reversible, may justify the use of more aggressive reperfusion or nervous tissue protection therapies.

Furthermore, because of the unique correlation between diffusion and temperature (Eq [19], Fig 13), diffusion MR imaging has also been proposed for the real-time and noninvasive monitoring of temperature (87). Noninvasive and nondestructive temperature imaging in biologic systems may be useful in many disciplines; for instance, in the study and monitoring of tissue interactions in surgical and medical laser procedures $(88,89)$. Another important application is clinical hyperthermia, which is becoming an increasingly used adjunctive treatment for cancer (90). Although promising results have been obtained, the clinical use of hyperthermia treatment for deeper-lying tissues is limited, mainly because of a lack of temperature control (91). The idea of using MR imaging was considered in earlier work (92-95) that exploited the temperature dependence of MR parameters, primarily that of T 1 of water $(92,94)$, which has been demonstrated in vitro for different biologic systems (96). Unfortunately, the relationship between $\mathrm{T} 1$ and temperature is not unique, mainly because of the mul- 


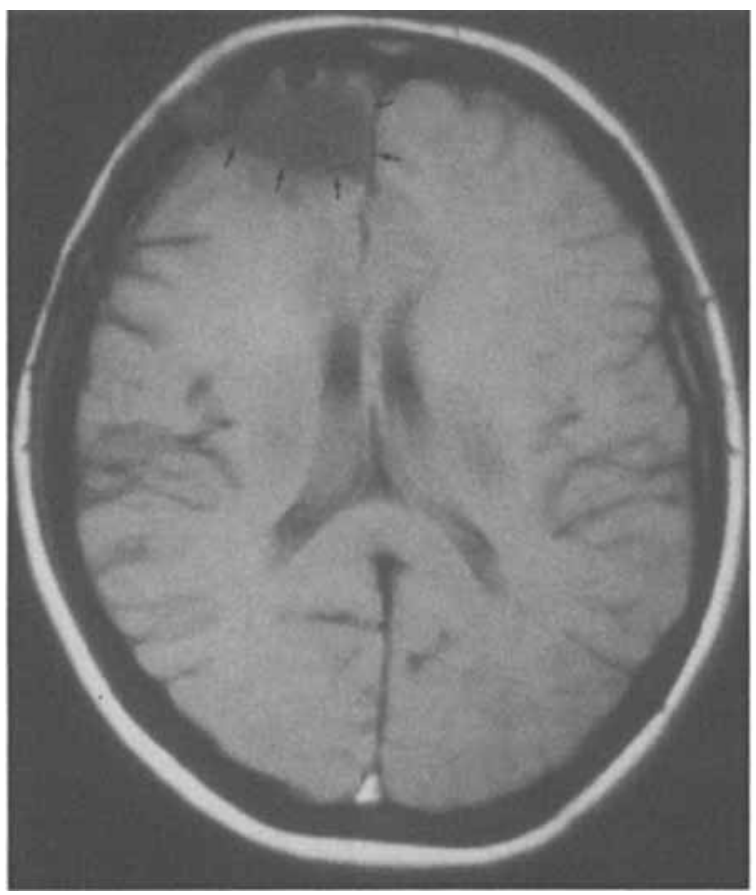

a.

Figure 17. Recurrent glioma after surgery. (a) Axial T1weighted image $(500 / 29)$ shows a porencephalic cavity (arrows). (b) Axial T2-weighted image $(1,700 / 140)$ shows the porencephalic cavity and edema with long T2s. The original tumor bed (arrows) does not provide specific information on tumor status. (c) IVIM image of the same section. Edema appears as a homogeneous area with a large ADC that is slightly smaller than the diffusion coefficient of pure water as found in the porencephalic cavity. The tumor bed looks inhomogeneous because of coexistence of regions of large and small ADCs, but on average, the tumor bed has a higher ADC than brain parenchyma. This inhomogeneity in the IVIM image associated with a large ADC is generally indicative of tumor activity. One part of the tumor bed (arrows) exhibits a very large ADC, which was ascribed to proliferation of microvessels and strongly suggested tumor recurrence (surgically proved), and which was not visible on $\mathrm{T} 1$ - and T2-weighted images. tifactorial nature of $\mathrm{T} 1(3,89,96,97)$ and the poor sensitivity of $\mathrm{T} 1$ to temperature. Molecular diffusion is a more sensitive temperature probe; the diffusion coefficients quantify thermal Brownian motion. According to Equation (19), the sensitivity of diffusion to temperature is on the order of $2.4 \%$ per degree Celsius (61). Obviously, the applications of temperature imaging are not limited to hyperthermia. Among potential applications are detection of possible temperature changes related to physiologic or pathologic status. Laser-tissue interaction studies in which power deposition and heating are key features may also benefit from this noninvasive temperature imaging method. Laser surgery may now be performed within an MR imaging unit (98). Similarly, first attempts have been made to design

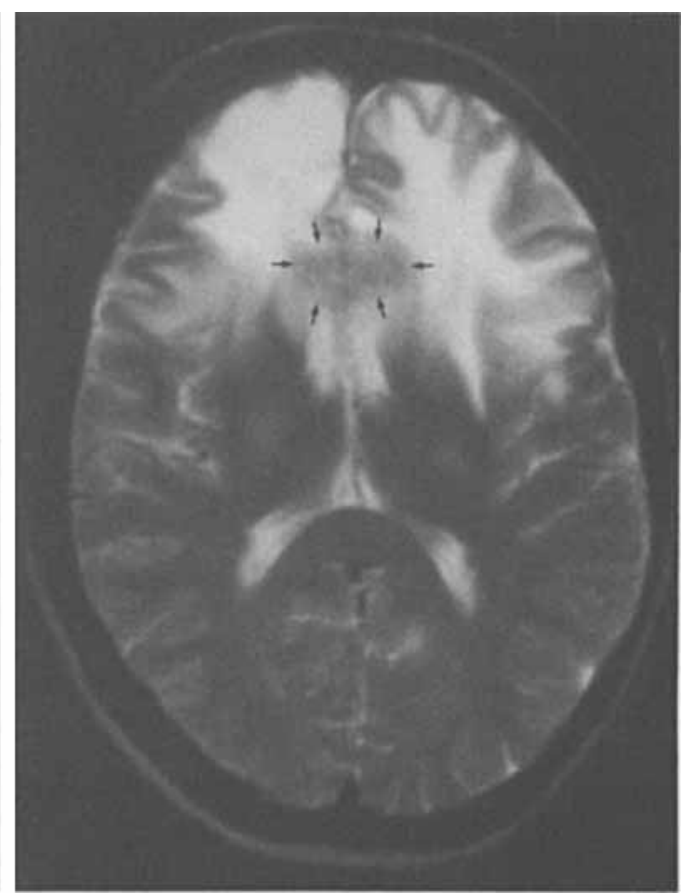

b.

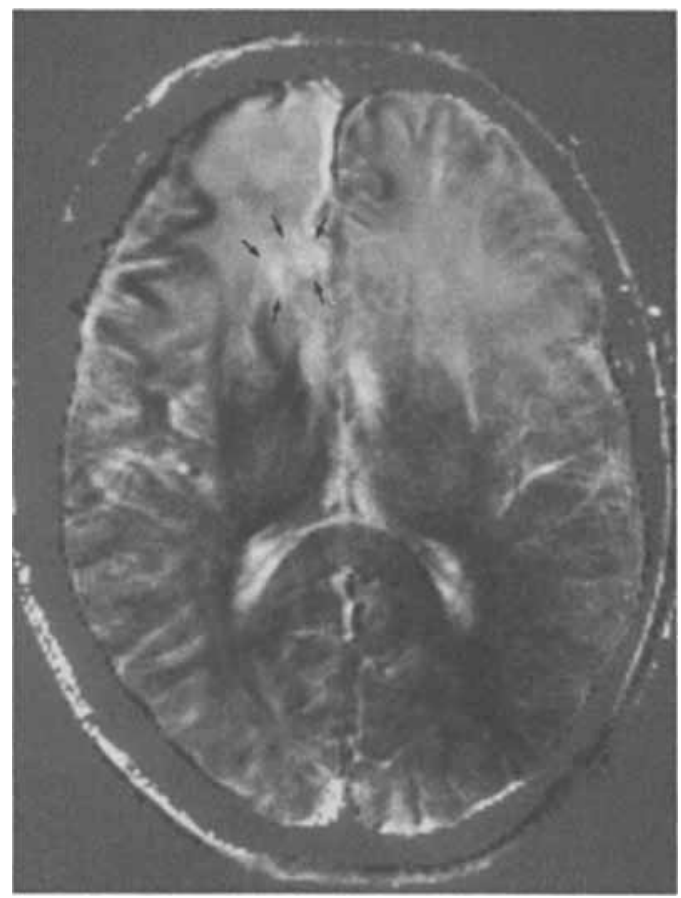

c.

a hyperthermia device (99) entirely compatible with an MR imaging unit (100). MR Imaging could therefore also become a useful device for monitoring ongoing therapeutic procedures.

Diffusion appears to be a "new" source of contrast in the field of MR imaging. Work remains to be 

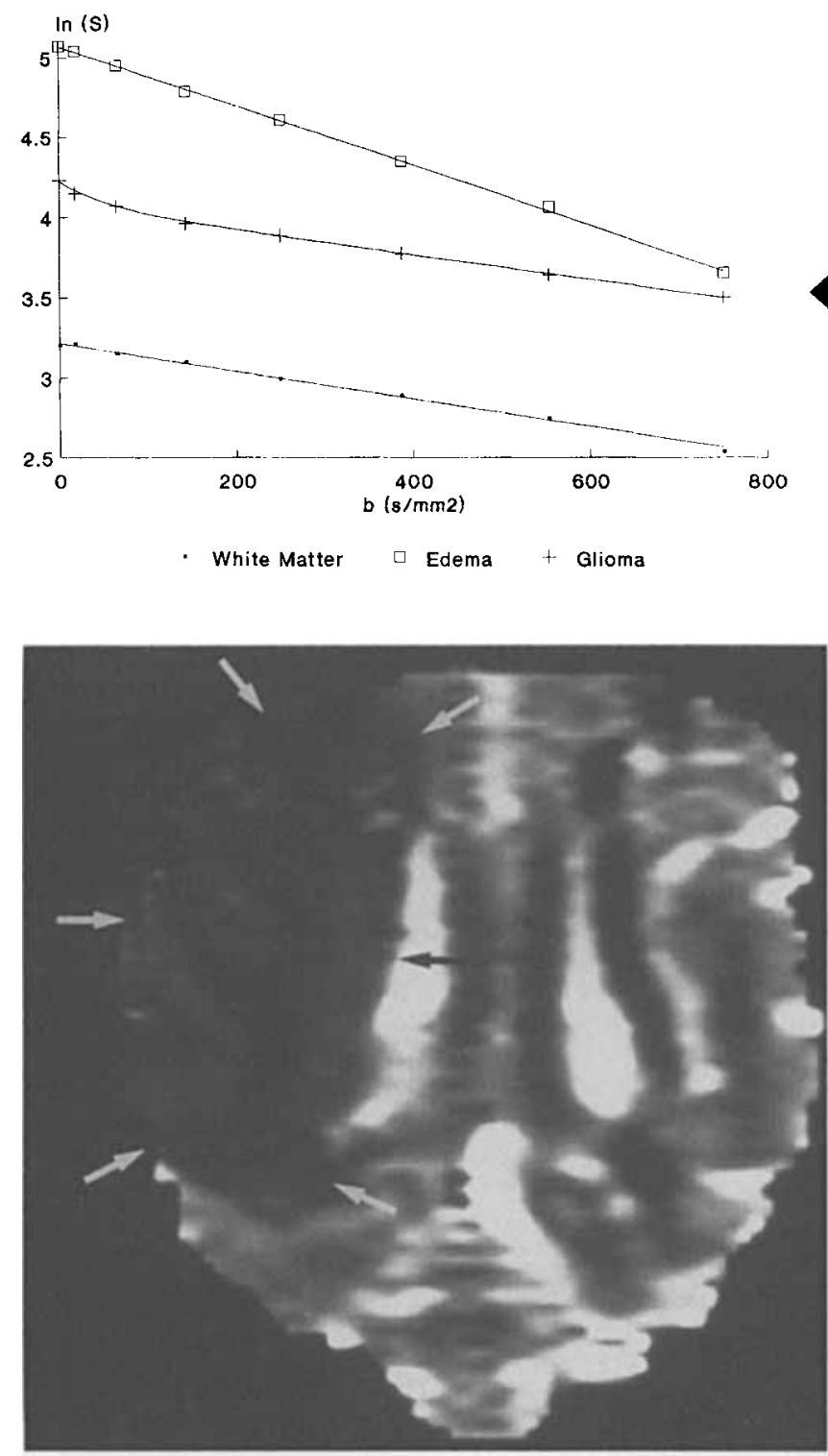

a.

Figure 19. Comparison of diffusion and T2-weighted images obtained in a cat brain 6 hours after occlusion of the left middle cerebral artery. These images were generated at $4.7 \mathrm{~T}$ with a STEAM sequence (TE of $80 \mathrm{msec}$, TR of $5 \mathrm{sec}$, diffusion time of $143 \mathrm{msec}$, diffusion gradients of $12.5 \mathrm{msec}$ duration and a gradient of $3.5 \mathrm{G} / \mathrm{cm}[35 \mathrm{mT} / \mathrm{m}]$ in the horizontal direction of the image (reprinted, with permission, from reference 79). (a) Calculated diffusion image. The ischemic region is clearly visible (arrows). The darkness of this area means that the ADC is dramatically decreased, reflecting a decrease of water mobility that occurs soon after the ischemic insult. (b) T2-weighted image of the same section shows only few abnormalities (increasd T2) limited to the cortex (arrows).

done to understand fully the significance and usefulness of this parameter for clinical applications. Diffusion is not per se an MR parameter, as has been mentioned, but MR imaging is the only available method to evaluate diffusion in vivo with accuracy and spatial resolution. It has been shown that there is no correlation between the diffusion coefficient and T1 and T2. T1 and T2 may be normal or elevated in diseased states while diffusion is lowered, as shown in early stroke.

By contrast, perfusion is a technically more chal-
Figure 18. Signal attenuation curves in a patient with a brain glioma. Both white matter and edema show straight lines, suggesting pure diffusion effects. The diffusion coefficient (slope) in edema $\left(D=1.86 \times 10^{-3} \mathrm{~mm}^{2} / \mathrm{sec}\right)$ is significantly larger than in white matter $\left(D=0.86 \times 10^{-3}\right.$ $\mathrm{mm}^{2} / \mathrm{sec}$ ), probably because of the presence of free interstitial water. In the tumor, the diffusion coefficient is decreased $\left(D=0.77 \times 10^{-3} \mathrm{~mm}^{2} / \mathrm{sec}\right)$. The curvature near the origin of the plot has been ascribed to perfusion $(f=$ $13 \%, D^{*}=29 \times 10^{-3} \mathrm{~mm}^{2} / \mathrm{sec}$.

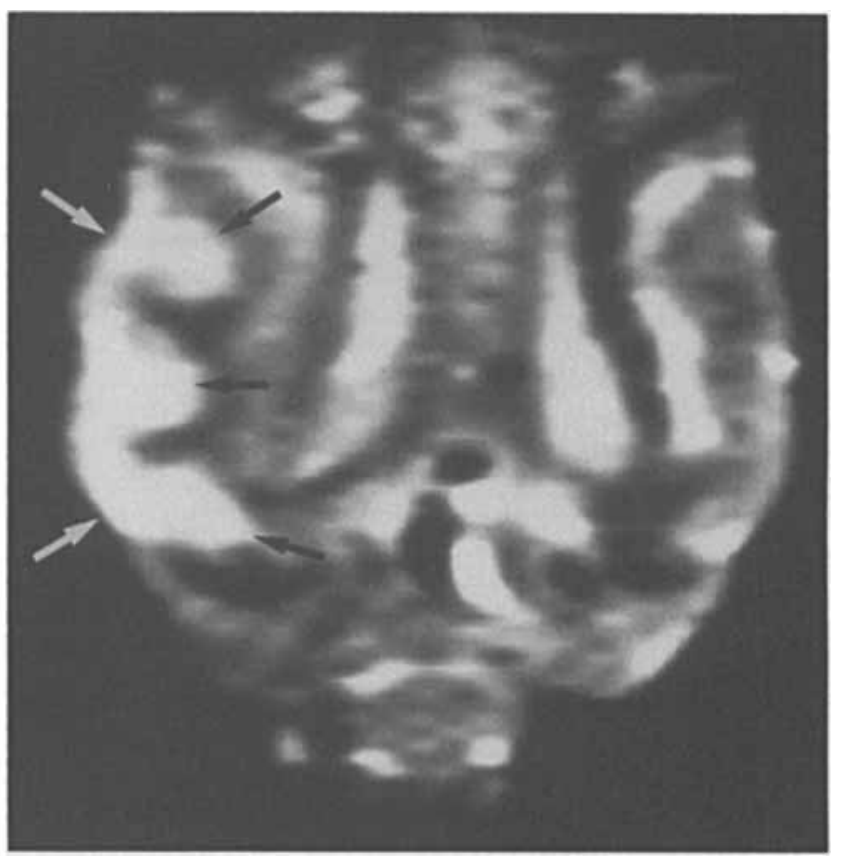


imaging methods can be useful if they demonstrate better spatial or temporal resolution or if they are easier to use. Perfusion MR imaging methods using gradient sensitization represent a fundamentally different approach to the concept of "perfusion." Perfusion includes both blood capillary microcirculation and blood-tissue exchanges. Classically, perfusion has been quantified in terms of milliliters per minute of blood per $100 \mathrm{~g}$ of tissue (ie, blood flow). With IVIM MR Imaging, however, perfusion is seen more as microcirculation and can be quantified in terms of active capillary density (milliliters of circulating blood per $100 \mathrm{~g}$ of tissue) derived from $b$ or average blood velocity (millimeters per second) derived from $D^{*}$. Further assumptions about capillary bed geometry are required in order to obtain results in milliliters per minute per $100 \mathrm{~g}$. However, if we accept that perfusion is independent within an organ (ie, does not depend on the volume of measurement), there must be a relationship between flow patterns and tissue delivery to satisfy the equation of continuity (56). This relationship makes the product $b \times D^{*}$ proportional to blood flow (milliliters per minute per $100 \mathrm{~g}$ of tissue). Therefore, the parameters accessible with gradient sensitization methods may bring new insights into microcirculation physiology that are not avallable with current techniques. For instance, it could be determined whether blood velocity or capillary volume are involved in blood flow changes in physiologic or pathologic conditions. That is why, despite technical difficulties, perfusion MR imaging by means of gradient sensitization may prove to be a useful source of important progress in our understanding of normal and abnormal tissue function.

"Perfusion-sensitized" images have also been obtained in clinical practice, mainly with the IVIM technique in brain (43). They have allowed highly perfused tumors to be identified by patterns different from those of poorly perfused tumors. Diffusion/perfusion images have also shown abnormalities that regular $\mathrm{T} 1$ - and $\mathrm{T} 2$-weighted images have failed to show. Work remains to be done to characterize how capillary blood flow is seen with gradient sensitization techniques and to fully establish the reliability of these techniques in obtaining accurate, reliable, and reproducible data. Perhaps the most exciting area is imaging of brain activity as it varies according to physiologic or pathologic conditions. Despite low spatial resolution, variations in cerebral blood flow with external stimulation have been shown with positron emission tomography. It would be fascinating to obtain such results with the high spatial and temporal resolution of perfusion MR imaging without the need for tracers or ionizing radiations.

\section{References}

1. Hahn EL. Spin-echoes. Phys Rev 1950: 80:580-594.

2. Singer JR. Blood flow rates by NMR measurements. Science 1959; 130:1652-1653.

3. Bottomley PA, Forster TH, Argersinger RE, Pfeifer LM. A review of normal tissue hydrogen NMR relaxation times and relaxation mechanisms from $1-100 \mathrm{MHz}$ : dependency on tissue type, NMR frequency, tempera- ture, species, excision, and age. Med Phys 1984; $11: 425-448$

4. Callaghan PT, Eccles CD. Diffusion-limited resolution in nuclear magnetic microscopy. J Magn Reson 1988; 78:1-8.

5. Le Bihan D, Breton E, Lallemand D, Grenier P, Cabanis EA, Laval-Jeantet $M$. MR imaging of intravoxel incoherent motions: application to diffusion and perfusion in neurologic disorders. Radiology 1986; $161: 401-407$

6. Jost $W$. Diffusion in solids, liquids, gases. New York: Academic Press. 1960.

7. Mills R. Self-diffusion in normal and heavy water in the range $1-45^{\circ}$. J Phys Chem 1973: 77:685-688.

8. Cooper RL, Chang DB. Young AC. Restricted diffusion in biophysical systems. Biophys J 1974; 14:161-177.

9. Stejskal EO. Use of spin-echoes in a pulsed magnetic field gradient to study anisotropic, restricted diffusion and flow. J Chem Phys 1965; 43:3597-3603.

10. Tanner JE, Stejskal EO. Restricted self-diffusion of protons in colloidal systems by the pulsed-gradient. spin-echo method. J Chem Phys 1968; 49:1768-1777.

11. Wayne RC, Cotts RM. Nuclear magnetic resonance study of diffusion in a bounded medium. Phys Rev 1966: 151:264-272.

12. Tanner JE. Transient diffusion in a system partitioned by permeable barriers: application to NMR measurements with a pulsed field gradient. J Chem Phys 1978; 69:1748-1754

13. Carr HY, Purcell EM. Effects of diffusion on free precession in nuclear magnetic resonance experiments. Phys Rev 1954; 94:630-638.

14. Woessner DE. Effects of diffusion in nuclear magnetic resonance spin-echo experiments. J Chem Phys 1961 ; 34:2057-2066.

15. Wesbey G, Moon K, Crooks L, Arakawa M, Brash R. Proton T2 reduction due to spin-diffusion through pulsed-gradients in spin-echo NMR imaging: imaging implications and applications. Magn Reson Med 1984; $1: 273-277$

16. Stejskal EO, Tanner JE. Spin diffusion measurements: spin echoes in the presence of a time-dependent field gradient. J Chem Phys 1965; 42:288-292.

17. Le Bihan $\mathrm{D}$, Breton $\mathrm{E}$. Imagerie de diffusion in-vivo par resonance magnetique nucleaire. CR Acad Sci (III) 1985; 301:1 109-1112.

18. Merboldt KD, Hanicke W, Frahm J. Self-diffusion NMR imaging using stimulated echoes. J Magn Reson $1985 ; 64: 479-486$.

19. Taylor DG, Bushell MC. The spatial mapping of translational diffusion coefficients by the NMR imaging technique. Phys Med Biol 1985; 30:345-349.

20. Wesbey GE, Moseley ME, Ehman RL. Translational molecular self-diffusion in magnetic resonance imaging: effects and applications. Invest Radiol 1984 19:491-498.

21. Kumar A, Welti D, Ernst RR. NMR Fourier zeugmatography. J Magn Reson 1975; 18:69-83.

22. Karlicek RF, Lowe IJ. A modified pulsed gradient technique for measuring diffusion in the presence of large background gradients. J Magn Reson 1980 ; 37:75-91.

23. von Meerwall $E$, Kamat $M$. Effect of residual field gradients on pulsed-gradient NMR diffusion measurements. J Magn Reson 1989; 83:308-323.

24. Ahn CB, Lee SV, Nalcioglu O, Cho ZH. An improved NMR diffusion coefficient imaging method using an optimized pulse sequence. Med Phys 1986; 13:789-793.

25. Thomsen $C$, Henriksen $O$, Ring $P$. In vivo measurement of water self-diffusion in the human brain by magnetic resonance imaging. Acta Radiol 1987; 28:353-361.

26. Moseley ME, Cohen Y, Kucharczyk J, et al. Diffusionweighted MR imaging of anisotropic water diffusion in cat central nervous system. Radiology $1990 ; 176: 439-$ 446.

27. Le Bihan D. Intravoxel incoherent motion imaging us- 
ing steady-state free precession. Magn Reson Med 1988: 7:346-351.

28. Le Bihan D. Turner R, MacFall JR. Effects of intravoxel incoherent motions (IVIM) in steady-state free precession (SSFP) imaging: application to molecular diffusion imaging. Magn Reson Med 1989; 10:324337.

29. Merboldt KD, Hanicke W, Gyngell ML, Frahm J, Bruhn H. Rapid NMR imaging of molecular self-diffusion usIng a modified CE-FAST sequence. J Magn Reson 1989; 82:115-121.

30. Merboldt KD, Bruhn H, Frahm J, Gyngell ML, Hanicke W, Deimling M. MRI of "diffusion" in the human brain: new results using a modified CE-FAST sequence. Magn Reson Med 1989; 9:423-429.

31. Turner R, Le Bihan D. Single-shot diffusion imaging at 2.0 Tesla. J Magn Reson $1990 ; 86: 445-452$.

32. Turner R, Le Bihan D, Majer J, Vavrek R, Hedges LK, Pekar J. Echo-planar imaging of intravoxel incoherent motions. Radiology 1990; 177:407-414.

33. Tanner JE. Use of the stimulated-echo in NMR diffusion studies. J Chem Phys 1970; 52:2523-2526.

34. MacFall JR, Le Bihan D. Comparison of stimulated echo, spin echo, and steady-state free precession pulse sequences for MR imaging apparent diffusion coefficients (abstr). Radiology 1988; 169(P):344.

35. Kayser R, Bartholdi E, Ernst RR. Diffusion and fieldgradient effects in NMR Fourier spectroscopy. J Chem Phys 1974; 60:2966-2979.

36. Wu EX, Buxton RB. Effect of diffusion on the steadystate magnetization with pulsed field gradients. J Magn Reson 1990 (in press)

37. Deimling $\mathbf{M}$, Mueller $\mathrm{E}$, Laub $\mathrm{G}$. Diffusion weighted imaging with turbo-FLASH (abstr). In: Book of abstracts: Society of Magnetic Resonance in Medicine 1990. Berkeley, Calif: Society of Magnetic Resonance in Medicine, $1990 ; 387$.

38. Perman WH, Gado M, Sandstrom JC. DPSF: snapshot flash diffusion/perfusion imaging (abstr). In: Book of abstracts: Society of Magnetic Resonance in Medicine 1990. Berkeley, Calif: Society of Magnetic Resonance in Medicine, $1990 ; 309$.

39. Haase A. Snapshot FLASH MRI: Applications to T1. T2, and chemical-shift imaging. Magn Reson Med 1990: 13:77-89.

40. Becker ED, Ferretti JA, Farrar TC. Driven equilibrium Fourier transform spectroscopy: a new method for nuclear magnetic resonance signal enhancement. J Am Chem Soc 1969; 91:7784-7785.

41. Edelman RR, Atkinson DJ. Turbosteam diffusion imaging (abstr). In: Book of abstracts: Society of Magnetic Resonance in Medicine 1990. Berkeley, Calif: Society of Magnetic Resonance in Medicine, 1990; 1285.

42. Merboldt KD, Hanicke W, Bruhn H, Gyngell ML, Frahm J. Diffusion-weighted high-speed STEAM MR imaging (abstr). In: Book of abstracts: Society of Magnetic Resonance in Medicine 1990. Berkeley, Calif: Society of Magnetic Resonance in Medicine, 1990; 1286.

43. Le Bihan D, Breton E, Lallemand D, Aubin ML, Vignaud J, Laval-Jeantet $M$. Separation of diffusion and perfusion in intravoxel incoherent motion (IVIM) MR imaging. Radiology 1988; 168:497-505

44. Chenevert TL, Brunberg JA, Pipe JG. Antsotropic diffusion within human white matter: demonstration with NMR techniques in vivo. Radiology 1990; 177:401-405.

45. Mansfield P. Multiplanar image formation using NMR spin echoes. J Phys Chem Solid State Phys 1977 10:L55-L58.

46. Avram HE, Crooks LE. Effect of self-diffusion on echoplanar imaging (abstr). In: Book of abstracts: Society of Magnetic Resonance in Medicine 1988. Berkeley, Calif: Society of Magnetic Resonance in Medicine, 1988; 80

47. Havseman AM, Stehling MK, Chapman B, et al. Improvements in snapshot nuclear magnetic resonance imaging. Br J Radiol 1988; 61:822-828.
48. Bryant DJ, Payne JA, Firmin DN, Longmore DB. Measurement of flow with NMR imaging using a gradient pulse and phase difference technique. J Comput Assist Tomogr 1984; 8:588-593.

49. Le Bihan D. Magnetic resonance imaging of perfusion Magn Reson Med 1990: 14:283-292.

50. Young IR, Hall AS, Bryant DJ, et al. Assessment of brain perfusion with MR imaging. J Comput Assist Tomogr 1988; 12:721-727

51. Ahn CB. Lee SY, Nalcioglu O, Cho ZH. The effects of random directional distributed flow in NMR imaging. Med Phys 1987: 14:43-48.

52. Maki JF, Cofer GP, Johnson GA. Separation of perfusion and diffusion effects in MR imaging (abstr). Radiology 1988: 169(P): 155

53. Xiang $9 S$. Nalcioglu $O$. New considerations in the measurement of perfusive flow by NMR (abstr). Magn Reson Imaging 1990; 8 (suppl 1):30.

54. Young IR, Coutts GA, Bryant DJ, Bydder GM. Use of ultralong echo time imaging to assist in measuring the velocity of perfused flow in vivo. Magn Reson Med 1989; 10:349-361.

55. Pawlik G, Rackl A, Bing RJ. Quantitative capillary topography and blood flow in the cerebral cortex of cats: an in-vivo microscopic study. Brain Res $1981 ; 208: 35$ 58.

56. Turner R. Perfusion studies and fast imaging. In: Rescigno A, Boicelli A, eds. Cerebral blood flow. New York: Plenum, 1988; 245-258

57. Turner R, Bowley RM. Passive screening of switched magnetic field gradients. J Phys E Sci Instrum 1986 19:876.

58. Mansfield P. Chapman B. Active magnetic screening of gradient coils in NMR imaging. J Magn Reson 1986 66:573-576

59. Roemer PB, Edelstein WA, Hickey JS. Self-shielded gradient colls (abstr). In: Book of abstracts: Society of Magnetic Resonance in Medicine 1986. Berkeley, Calif Society of Magnetic Resonance in Medicine, 1986; 1067.

60. Hayes CE, Edelstein WA, Schenck JF, Mueller OM, Eash M. An efficient, highly homogeneous radiofre. quency coil for whole-body NMR imaging at $1.5 \mathrm{~T}$. J Magn Reson 1985; 63:622-628.

61. Simpson JH, Carr HY. Diffusion and nuclear spin relaxation in water. Phys Rev 1958: $111: 1201-1202$.

62. Chang DC, Rorschach HE, Nichols BL, Hazlewood CF Implications of diffusion coefficient measurements for the structure of cellular water. Ann NY Acad Sci 1973 204:434-443

63. Lorentz CH, Pickens DR, Puffer DB, Price RR. Diffusion/perfusion phantom experiments. In: Syllabus of the Meeting on Future Directions in MRI of Diffusion and Microcirculation. Berkeley, Calif: Society of Magnetic Resonance in Medicine, 1990; 123.

64. Turner R, Le Bihan D, Maier J, Vavrek R, Baich J. Echo-planar IVIM imaging of the body: application to kidney (abstr). In: Book of abstracts: Society of Magnetic Resonance in Medicine 1990. Berkeley, Calif: Society of Magnetic Resonance in Medicine, 1990: 1136.

65. Feinberg DA, Mark AS. Human brain motion and cerebrospinal fluid circulation demonstrated with MR velocity imaging. Radiology 1987; 163:793-799.

66. Marquardt DW. An algorithm for least-squares estimation of nonlinear parameters. J Soc Ind Appl Math. 1963; $11: 431$.

67. Bevington PR. Data reduction and error analysis for the physical sciences. New York: McGraw-Hill, 1969.

68. Rubinstein RY. Simulation and the Monte Carlo method. New York: Wiley, 1981.

69. Pekar J, Moonen CTW, van Zijl PCM. On the precision of diffusion/perfusion imaging by gradient sensitization. Magn Reson Med (in press).

70. Paulson OB. Hertz MM, Bolwig TG, Lassen NA. Filtra. tion and diffusion of water across the blood-brain barrier in man. Microvasc Res 1977; 13:1 13-124. 
71. Feinberg DA, Crooks LE, Sheldon $P$, et al. Magnetic resonance imaging of the velocity vector components of fluid flow. Magn Reson Med 1985; 2:555-566.

72. Hansen JR. Pulsed NMR study of water mobility in muscle and brain tissue. Blochim Biophys Acta 1971; 230:482-486.

73. Brownstein KR, Tarr CE. Importance of classical diffusion in NMR studies in biological cells. Phys Rev 1979; A 19:2446-2453.

74. Moonen CTW, van Zijl PCM, Le Bihan D, DesPres D. In vivo NMR diffusion spectroscopy: ${ }^{31} \mathrm{P}$ application to phosphorus metabolites in muscle. Magn Reson Med 1990: 13:467-477.

75. Finkelstein A. Water movement through lipid bilayers, pores, and plasma membranes: theory and reality. New York: Wiley, 1987

76. Koenig SH, Brown RD, Spiller M, Lundbom N. Relaxometry of brain: why white matter appears bright in MRI. Magn Reson Med 1990; 14:482-495.

77. Cleveland GG, Chang DC, Hazlewood CF. Nuclear magnetic resonance measurement of skeletal muscle: anisotropy of the diffusion coefficient of the intracellular water. Blophys J 1976; 16:1043-1053.

78. Le Bihan D, Turner R, Douek $P$. Is water diffusion restricted in human brain? (abstr). In: Book of abstracts: Society of Magnetic Resonance in Medicine 1990. Berkeley, Calif: Society of Magnetic Resonance in Medicine. 1990: 377.

79. Moonen CTW, de Vleeschouwer MHM, DesPres D, van Zijl PCM, Pekar J. Restricted and anisotropic displacement of water in healthy cat brain and in stroke by NMR diffusion imaging (abstr). In: Book of abstracts: Society of Magnetic Resonance in Medicine 1990. Berkeley. Calif: Society of Magnetic Resonance in Medicine, 1990; 1121 .

80. van Zijl PCM, Moonen CTW, Kaplan O, Faustino P, Cohen JS. Complete separation of intracellular and extracellular information in NMR spectra of perfused cell cultures (abstr). In: Book of abstracts: Society of Magnetic Resonance in Medicine 1990. Berkeley, Calif: Society of Magnetic Resonance in Medicine, 1990; 851.

81. Tsuruda JS, Chew WM, Moseley ME, Norman D. Diffusion-weighted MR imaging of the brain: value of differentiating between extraaxial cysts and epidermoid tumors. AJNR 1990; 11:925-931.

82. Haimes A, Zimmerman R, Morgello S, et al. MR imaging of brain abscesses. AJNR 1989; 10:279-291.

83. Le Bihan D, Breton E, Aubin ML. Study of cerebrospinal fluid dynamics by MRI of intravoxel incoherent motions (IVIM). J Neuroradiol 1987; 14:388-395.

84. Moseley ME, Kucharczyk J, Mintorovitch J, et al. Diffusion-weighted MR imaging of acute stroke: correlation with T2-weighted and magnetic susceptibility-enhanced MR imaging in cats. AJNR 1990; 11:423-429.

85. Garcia JH, Anderson ML. Physiopathology of cerebral ischemia. CRC Crit Rev Neurobiol 1989; 4:303-324.

86. Le Bihan D, Berry I, Gelbert F, et al. MR imaging of intravoxel incoherent motions in ischemic and malformative cerebral diseases (abstr). Radiology 1987; 165(P):303.

87. Le Bihan D, Delannoy J, Levin R. Non-invasive temperature mapping using magnetic resonance imaging of molecular diffusion: application to hyperthermia.
Radiology 1989; 171:853-857.

88. Svaasand LO, Boerslid T, Oeveraasen $M$. Thermal and optical properties of living tissue: application to laser induced hyperthermia. Laser Surg Med 1985: 5:589602.

89. Jolesz FA, Bleier AR, Jakab P, Ruenzel PW, Huttl K, Jako GJ. MR Imaging of laser-tissue interactions. Radiology 1988; 168:249-253.

90. Hahn GM. Hyperthermia and cancer. 2nd ed. New York: Plenum, 1982

91. Cetas TC. Will thermometric tomography become practical for hyperthermia treatment monitoring? Cancer Res 1984; 44(suppl):4805-4808.

92. Dickinson RJ, Hall AS, Hind AJ, Young IR. Measurement of changes in tissue temperature using MR Imaging. J Comput Assist Tomogr 1986; 10:468-472.

93. Knuttel B, Juretschke HP. Temperature measurements by nuclear magnetic resonance and its possible use as a means of in vivo noninvasive temperature measurement and for hyperthermia treatment assessment. Recent Results Cancer Res 1986; 101:109-1 18.

94. Parker DL, Smith V, Sheldon P, Crooks LE, Fussel L. Temperature distribution measurements in two-dimensional NMR imaging. Med Phys 1983: 10:321325.

95. Tanaka H, Eno K, Kato H, Ishida T. Possible application of non-invasive thermometry for hyperthermia using NMR. Nippon Acta Radiol 1981; 41:897-899.

96. Lewa CJ, Majeska $Z$. Temperature relationships of proton spin-lattice relaxation time $\mathrm{T} 1$ in biological tissues. Bull Cancer (Paris) 1980; 67:525-530.

97. Fung BM. Proton and deuteron relaxation of muscle water over wide ranges of resonance frequencies. Biophys J 1977; 18:235-239.

98. Jolesz FA, Higuchi N, Bleier AR, Jakab P. MRI of laser effects on tissue (abstr). Magn Reson Imaging 1990; 8 (suppl 1): 156.

99. Turner PF. Mini-annular phased array for limb hyperthermia. IEEE Trans Microwave Theory Technol 1986; 34:508-513.

100. Delannoy J, Le Bihan D, Hoult D, Levin R. Hyperthermia system combined with an MRI unit. Med Phys 1990; 17:855-860.

101. Barranco D, Sutton LN, Florin S, et al. Use of ${ }^{19} F$ NMR spectroscopy for measurement of cerebral blood flow: a comparison study using microspheres. J Cereb Blood Flow Metab 1989: 9:886-891.

102. Eleff SM, Snall MD, Ligetti L, et al. Concurrent measurements of cerebral blood flow, sodium, lactate, and high-energy phosphate metabolism using ${ }^{19} \mathrm{~F},{ }^{23} \mathrm{Na},{ }^{1} \mathrm{H}$, and ${ }^{31} \mathrm{P}$ NMR spectroscopy. Magn Reson Med 1988 ; 7:412-424.

103. Ewing JR, Branch CA, Helpern JA, Smith MB, Butt SM, Welch KM. Cerebral blood flow measured by NMR indicator dilution in cats. Stroke 1989; 20:259-267.

104. Ackerman JJH, Ewy CS, Becker NN, Shalwitz RA. Deuterium nuclear magnetic resonance measurements of blood flow and tissue perfusion employing ${ }^{2} \mathrm{H}_{2} \mathrm{O}$ as a freely diffusible trace. Proc Natl Acad Sci USA 1987; 84:4099-4102.

105. Rosen BR, Belliveau JW, Chien D. Perfusion imaging by nuclear magnetic resonance. Magn Reson Q 1990; $5: 263-281$ 